\title{
The Effectiveness of Psychological Treatments in Women with Breast Cancer: A Systematic Review and Meta-Analysis
}

\author{
Angela Guarino ${ }^{1, *}$, Cristina Polini ${ }^{2}$, Giuseppe Forte ${ }^{1}\left(\mathbb{D}\right.$, Francesca Favieri $^{1}{ }^{\mathbb{D}}$, \\ Ilaria Boncompagni ${ }^{2}$ and Maria Casagrande ${ }^{2}$ \\ 1 Department of Psychology, “Sapienza” University of Rome, 00185 Rome, Italy; g.forte@uniroma1.it (G.F.); \\ francesca.favieri@uniroma1.it (F.F.) \\ 2 Department of Dynamic and Clinical Psychology, "Sapienza" University of Rome, 00185 Rome, Italy; \\ cristina.polini@gmail.com (C.P.); ilaria.boncompagni@uniroma1.it (I.B.); \\ maria.casagrande@uniroma1.it (M.C.) \\ * Correspondence: angela.guarino@uniroma1.it
}

Received: 12 December 2019; Accepted: 9 January 2020; Published: 12 January 2020

\begin{abstract}
Breast cancer is the most prevalent oncological disease among women, and it represents the second oncological cause of death. Many studies have considered the quality of life in people with breast cancer because this condition has high comorbidity with mental distress, anxiety, affective disorders and depression. Psychological interventions can reduce the stressful consequences of both the diagnosis and the medical treatments of breast cancer. However, different methods (e.g., group or individual therapy) and focus (e.g., improving personal skills or increasing emotional well-being) do not help to identify which type of psychological therapy can be more effective in improving quality of life in patients with breast cancer. This study was aimed to systematically review and compare, by means of a meta-analysis, the efficacy of cognitive behavioural, supportive-expressive or psycho-educational treatments in women with breast cancer, focusing on anxiety, depression, mood and quality of life as outcomes. The PRISMA statement was adopted. MEDLINE, PsycINFO, PUBMED and PsycArticles databases were used, and reference lists were examined for additional publications. In the selection of the articles were included studies considering women between 18 and 65 years who were diagnosed with breast cancer at any stage and under any treatment, and who underwent psychological group interventions. At the end of the systematic review, 45 studies met all inclusion criteria and were analysed in the meta-analysis. The overall effect size was medium, especially considering cognitive behavioural therapy and psycho-educational treatments. However, the studies are characterised by high methodological heterogeneity. Despite some limitations, this review and meta-analysis partially confirm the efficiency of cognitive-behavioural and psycho-educational therapies in the improvement of well-being in women with breast cancer.
\end{abstract}

Keywords: breast cancer; psychological treatment; cognitive behavioural therapy; psycho-educational therapy; supportive expressive therapy; anxiety; depression; mood; quality of life

\section{Introduction}

Breast cancer is the most prevalent oncological disease in women, with a lifetime incidence ratio of one in eight $[1,2]$. It represents the second oncological cause of death [3,4] and twenty-five per cent of all new cancer diagnoses in women, with a higher incidence in the age range from 55 to 64 years $[4,5]$. Despite the growing and ageing of the global population, and the increasing number of new breast cancer diagnoses, survival rates have been improved due to advancements in the assessment and treatment [1]. 
Lifespan consequences and quality of life in women with breast cancer have been the most studied [6,7].

Breast cancer shows high a comorbidity rate, with mental distress [8,9], anxiety and affective disorders $[8,10]$, depression [11,12], and chronic fatigue and decreased social interactions being common responses to breast cancer diagnosis and treatment [13-15]. Moreover, women with a primary breast cancer diagnosis remain vulnerable to psychological disorders for many years [16-19], highlighting the high impact of this medical condition in the quality of life of the patients.

Psychological interventions could reduce the distress consequent to both the diagnosis and medical treatment of breast cancer. However, the studies assessing the effectiveness of psychological therapies are characterised by a heterogeneity of methods (e.g., group or individual therapy) and focuses (e.g., improving personal skills or increasing emotional well-being).

Among the different psychological approaches, cognitive behavioural therapy (CBT) is focused on improving the affective state and coping with the disease $[20,21]$ through the modification of maladaptive cognitive schemas and the diminution of personal distress. CBT adopts different strategies (e.g., relaxation training), and it appears to be effective in reducing levels of anxiety and depression [22,23], restructuring negative automatic cognitive schemas [24], and increasing optimism and positive thought $[3,25,26]$, which help to improve the quality of life of patients with breast cancer $[6,7]$.

Other conventional psychological approaches in the treatment of women with breast cancer are the supportive-expressive and psycho-educational therapies, mainly characterised by group interventions focused on the improvement of personal well-being through the increase of positive affectivity and emotional states [27-29]. Supportive-expressive group therapy (SET) is a cognitive emotion-focused therapy aimed at promoting the development of social support and emotional expression and the examination of existential concerns [30]. SET appears to reduce emotional distress related to breast cancer $[27,30]$. Psycho-educational therapy (PET) is an interdisciplinary approach, including an educational program (e.g., providing adequate knowledge concerning the disease and its related treatments) and a psychological intervention (e.g., providing affective and cognitive skills to elaborate the experience related to the illness) [31]. Several studies have shown the efficacy of PET in improving adaptive coping strategies and quality of life, and in reducing the psychopathological symptomatology (as depression and anxiety). It also allows increasing both the treatment compliance and self-efficacy in women with breast cancer [32-34].

Scientific evidence (for a review see: [35,36]) confirmed the effectiveness of all these approaches, showing high positive effects in reducing anxiety and depression and improving quality of life. Moreover, these effects are maintained both in short- and long-term follow-ups [35,37,38].

Although previous reviews [39-43] have analysed this topic and highlighted how psychological interventions can ameliorate the quality of life of women with breast cancer, it is still unclear which intervention is the most effective. Generally, single studies and reviews have focused on a specific or limited number of psychological outcomes. However, acquiring information about several outcomes could be useful in order to both define the effectiveness of a specific intervention on the psychological well-being of patients and to develop more functional treatments. Additionally, there are a limited number of studies comparing different approaches. The aim of this study is therefore to systematically review and compare, through a meta-analysis, the effectiveness of different (cognitive behavioural, supportive-expressive, and psycho-educational) group therapies in women with breast cancer, focusing on different psychological outcomes (anxiety, depression, mood, and quality of life) as an expression of patients' psychological well-being. It was hypothesised that there would be a general efficacy of all these psychological interventions in patients who underwent these group treatments compared to control groups. Moreover, we analysed the differences in the effectiveness of these types of intervention, considering each psychological outcome. 


\section{Methods}

\subsection{Method}

The Preferred Reporting Items for Systematic Reviews and Meta-Analyses (PRISMA) statements $[44,45]$ were adopted. Registration of the protocol was not planned.

\subsection{Research Strategies}

Two independent researchers (I.B, C.P.) conducted four electronic bibliographic database searches (PsychINFO, PubMed, Medline, PsycArticles) of the studies testing psychological interventions in women with breast cancer.

A list of keywords and MeSH terms was generated to identify studies falling within three key search strategies: 1. Terms related to breast cancer: "breast cancer", "breast neoplasm"; 2. Terms related to psychological intervention: "group psychotherapy", "supportive psychotherapy" "cognitive behaviour therapy", "expressive psychotherapy", "psycho-education *"; 3 . Terms related to outcomes: "quality of life", "anxiety", "depression", "distress", "emotional states".

Restrictions were made, limiting the research to academic publications with English full-text until February 2019. Additionally, the bibliographical references of retrieved articles, reviews, and meta-analyses were screened to assess whether they contained relevant studies to include in the review.

\subsection{Eligibility Criteria}

The studies were eligible for review if they included women between the ages of 18 and 65 who were diagnosed with breast cancer at any stage and under any treatment, and who were submitted to one psychological intervention. Specifically, the principal inclusion criteria were: (1) studies comparing a group who underwent psychological treatment and a control group with the same pathology but not subjected to the intervention (they received alternative intervention, usual care or waitlist); (2) studies which considered psycho-educational treatment (PET), cognitive behavioural therapy (CBT) or supportive-expressive group therapy (SET) aimed at improving quality of life and mood and to reduce anxiety and depression; (3) studies which adopted randomized controlled design and non-randomized controlled design were both included.

Exclusion criteria were: (1) treatments based on online or telephone interventions, intensive residential interventions, or single intensive session interventions; (2) studies considering individual treatment; (3) studies that compared the effectiveness of group interventions with individual treatment; (4) studies with a single-group pre-post comparison.

No restrictions about the provider of the intervention (e.g., oncologist, psychologist, psychiatrist, social worker, nurse, volunteer, or other) or length of follow-up were inserted. Any standardised screening or diagnostic measure could determine the outcome. Studies presenting composite results, methodological criticisms and those that did not report essential data were excluded.

\subsection{Study Selection}

After the removal of duplicates, the initial assessment of eligibility was based on titles and abstracts. Disagreement in selection was resolved by consulting supervisors (A.G. and M.C.). Two authors (G.F., F.F.) independently examined full texts to confirm the suitability of the studies for following qualitative and quantitative synthesis.

\subsection{Data Collection}

According to the PICOS approach (Population, Intervention, Comparator, Outcomes and Study Design) [44], the main information of each study, such as the author(s) and year of publication, size, and characteristics of the sample (cancer stage, condition during treatment), control and 
intervention groups conditions, provider type, total intervention time and psychological screening tools, were extracted.

Table 1 reports the data.

\subsection{Data Synthesis}

For the quantitative analyses, pre- and post-intervention means and standard deviations of the determined psychological variables were extracted.

\subsection{Qualitative Assessment}

The methodological quality of studies was assessed using the criteria from the Cochrane Handbook for Systematic Review [46] modified ad hoc according to the aims of this systematic review. The following items were included: use of the randomisation process, including allocation of random sequence (selection bias), selection of sample or baseline imbalances of sample group size (selection bias), use of appropriate tasks for the analysis of the psychological variables considered (detection bias), incomplete outcome data (attrition bias), selective reporting (reporting bias) and other biases.

The quality was categorised as unclear/low/high risk of bias ("+" for low risk of bias, "-" for high risk of bias and "?" for unclear risk of bias).

The overall risk of bias was defined according to the level of risk in the various domains. If a study showed a high risk of bias in at least one domain and showed unclear risk in more domains, it was classified as having a high risk of bias; low risk of bias was chosen if all the domains presented a low risk of bias. If a study showed an unclear risk in at least one domain, the study was considered to have some concern of risk of bias.

\subsection{Quantitative Analysis}

The mean effect size (ES) (Cohen's d) and the 95\% confidence interval (CI) for psychological outcomes were computed. Effect size was calculated by considering mean scores of intervention and control groups.

The interpretation of the ES was small (lower than 0.2), medium (between 0.21 and 0.5), and large (higher than 0.8) [47]. Given that the effect estimates from multiple studies show more variance than the effect drawn from a single population, the random effects model (REML) was adopted to estimate effect size, using the Sidik-Jonkman estimator and the Hartung-Knapp-Sidik-Jonkman (HKSJ) method [48]. Heterogeneity between studies was estimated using the $\mathrm{I}^{2}$, and $\mathrm{t}$ was considered as low $(25 \%)$, moderate $(50 \%)$, and high $(75 \%)$ [ 49 ].

Forest plots were used to display the overall ES and weight in percentage, which indicated the influence of the study. Subgroup analyses were conducted to determine which characteristics of both sample and intervention influenced the ES. This procedure also allowed the identification of effects determined by methodological characteristics of the studies (the type of provider, duration of intervention, control condition and inclusion criteria; see Table 1).

All analyses were carried out with R Studio. 
Table 1. Information about the studies included in the meta-analysis. For each study, the following variables are reported: the outcome and the instruments (Tools) utilised to evaluate it; the sample size (n); the Intervention Type; the Intervention Duration; the Control Condition; the Inclusion Criteria; the Provider; and the Stage of Cancer.

\begin{tabular}{|c|c|c|c|c|c|c|c|c|c|}
\hline Variables & Tools & Study & $\begin{array}{c}n \\
\text { (Treatment/Control) }\end{array}$ & $\begin{array}{l}\text { Intervention } \\
\text { Type }\end{array}$ & $\begin{array}{l}\text { Intervention } \\
\text { Duration }\end{array}$ & $\begin{array}{l}\text { Control } \\
\text { Condition }\end{array}$ & $\begin{array}{c}\text { Inclusion } \\
\text { Criteria }\end{array}$ & Provider & $\begin{array}{l}\text { Stage of } \\
\text { Cancer }\end{array}$ \\
\hline \multirow{10}{*}{ Anxiety } & HADS & Kissane et al. [24] & $145 / 141$ & $\begin{array}{c}\text { CBT + } \\
\text { Relaxation } \\
\text { Lessons }\end{array}$ & 20 weeks & $\begin{array}{l}\text { Relaxation } \\
\text { Lessons }\end{array}$ & $\begin{array}{l}\text { Inpatient } \\
\text { Treatment }\end{array}$ & Two Therapists & I; II \\
\hline & STAI & Dolbeault et al. [32] & $85 / 93$ & PET & 8 weeks & Wait List & $\begin{array}{c}\text { After } \\
\text { Treatment }\end{array}$ & Two Therapists & I; II; III \\
\hline & HADS & Groarke et al. [50] & $102 / 120$ & CB-CBSM & 5 weeks & Usual Care & $\begin{array}{c}\text { After } \\
\text { Treatment }\end{array}$ & $\begin{array}{c}\text { Clinical } \\
\text { Psychologist }\end{array}$ & From 0 to IV \\
\hline & HADS & Ho et al. [30] & $47 / 51$ & SET & 8 weeks & Social Support & $\begin{array}{c}\text { After } \\
\text { Treatment }\end{array}$ & Therapist & I; II; III \\
\hline & PAI & Jang et al. [51] & $12 / 11$ & CBT-MBSR & 12 weeks & Wait List & $\begin{array}{c}\text { After } \\
\text { Treatment }\end{array}$ & $\begin{array}{l}\text { Mental Health } \\
\text { Specialist }\end{array}$ & From 0 to III \\
\hline & HADS & Johannsen et al. [52] & $46 / 61$ & CBT-MBSR & 8 weeks & Wait List & $\begin{array}{c}\text { After } \\
\text { Treatment }\end{array}$ & $\begin{array}{l}\text { Mindfulness } \\
\text { Instructor }\end{array}$ & Not Reported \\
\hline & STAI & Lengacher et al. [53] & $152 / 147$ & CBT-MBSR & 6 weeks & Usual Care & $\begin{array}{c}\text { After } \\
\text { Treatment }\end{array}$ & $\begin{array}{c}\text { Clinical } \\
\text { Psychologist }\end{array}$ & From 0 to III \\
\hline & STAI & Reich et al. [54] & $159 / 152$ & CBT-MBSR & 6 weeks & Usual Care & $\begin{array}{c}\text { After } \\
\text { Treatment }\end{array}$ & $\begin{array}{c}\text { Clinical } \\
\text { Psychologist }\end{array}$ & From 0 to III \\
\hline & STAI & Zhang et al. [55] & $28 / 30$ & CBT-MBSR & 8 weeks & Usual Care & $\begin{array}{c}\text { After } \\
\text { Treatment }\end{array}$ & $\begin{array}{l}\text { Psychologist, } \\
\text { Expert in } \\
\text { Mindfulness }\end{array}$ & I; II; III \\
\hline & HADS & $\begin{array}{c}\text { Kenne Sarenmalm et } \\
\text { al. [56] }\end{array}$ & $62 / 52$ & CBT-MBSR & 8 weeks & Non-MBSR & $\begin{array}{c}\text { After } \\
\text { Treatment }\end{array}$ & $\begin{array}{l}\text { Mindfulness } \\
\text { Instructor }\end{array}$ & Not Reported \\
\hline
\end{tabular}


Table 1. Cont.

\begin{tabular}{|c|c|c|c|c|c|c|c|c|c|}
\hline Variables & Tools & Study & $\begin{array}{c}n \\
\text { (Treatment/Control) }\end{array}$ & $\begin{array}{l}\text { Intervention } \\
\text { Type }\end{array}$ & $\begin{array}{l}\text { Intervention } \\
\text { Duration }\end{array}$ & $\begin{array}{l}\text { Control } \\
\text { Condition }\end{array}$ & $\begin{array}{c}\text { Inclusion } \\
\text { Criteria }\end{array}$ & Provider & $\begin{array}{l}\text { Stage of } \\
\text { Cancer }\end{array}$ \\
\hline \multirow{12}{*}{ Depression } & HADS & Kissane et al. [24] & $145 / 141$ & $\begin{array}{c}\text { CBT + } \\
\text { Relaxation } \\
\text { Lessons }\end{array}$ & 20 weeks & $\begin{array}{l}\text { Relaxation } \\
\text { Lessons }\end{array}$ & $\begin{array}{l}\text { Inpatient } \\
\text { Treatment }\end{array}$ & Two Therapists & I; II \\
\hline & BDI & Kissane et al. [57] & $115 / 56$ & SET & 13 weeks & $\begin{array}{l}\text { Relaxation } \\
\text { Lessons }\end{array}$ & $\begin{array}{c}\text { After } \\
\text { Treatment }\end{array}$ & Therapist & IV \\
\hline & HADS & Groarke et al. [50] & $102 / 120$ & CBT-CBSM & 5 weeks & Usual Care & $\begin{array}{c}\text { After } \\
\text { Treatment }\end{array}$ & $\begin{array}{c}\text { Clinical } \\
\text { Psychologist }\end{array}$ & From 0 to IV \\
\hline & CES-D & Bower et al. [58] & $32 / 33$ & CBT-MAP & 6 weeks & Wait List & $\begin{array}{c}\text { After } \\
\text { Treatment }\end{array}$ & Not Reported & From 0 to III \\
\hline & FACT & Lechner et al. [59] & $57 / 57$ & CBT-CBSM & 10 weeks & $\begin{array}{l}\text { Educational } \\
\text { Program }\end{array}$ & $\begin{array}{c}\text { After } \\
\text { Treatment }\end{array}$ & $\begin{array}{c}\text { Clinical } \\
\text { Psychologist }\end{array}$ & From 0 to IV \\
\hline & CES-D & Dodds et al. [60] & $16 / 12$ & CBT-CDCT & 16 weeks & Wait List & $\begin{array}{c}\text { After } \\
\text { Treatment }\end{array}$ & $\begin{array}{l}\text { Clinically Trained } \\
\text { PhD and Social } \\
\text { Work Researcher }\end{array}$ & Not Reported \\
\hline & HADS & Ho et al. [30] & $47 / 51$ & SET & 8 weeks & Social Support & $\begin{array}{c}\text { After } \\
\text { Treatment }\end{array}$ & Therapist & I; II; III \\
\hline & PAI & Jang et al. [51] & $12 / 11$ & CBT-MBSR & 12 weeks & Wait List & $\begin{array}{c}\text { After } \\
\text { Treatment }\end{array}$ & $\begin{array}{l}\text { Mental Health } \\
\text { Specialist }\end{array}$ & From 0 to III \\
\hline & CES-D & Lengacher et al. [53] & $152 / 147$ & CBT-MBSR & 6 weeks & Usual Care & $\begin{array}{c}\text { After } \\
\text { Treatment }\end{array}$ & $\begin{array}{c}\text { Clinical } \\
\text { Psychologist }\end{array}$ & From 0 to III \\
\hline & CES-D & Reich et al. [54] & $154 / 146$ & CBT-MBSR & 6 weeks & Usual Care & $\begin{array}{c}\text { After } \\
\text { Treatment }\end{array}$ & $\begin{array}{c}\text { Clinical } \\
\text { Psychologist }\end{array}$ & From 0 to III \\
\hline & CES-D & Boyle et al. [61] & $35 / 30$ & CBT-MAP & 6 weeks & Wait List & $\begin{array}{c}\text { After } \\
\text { Treatment }\end{array}$ & Not Reported & From 0 to III \\
\hline & HADS & $\begin{array}{c}\text { Kenne Sarenmalm et } \\
\text { al. [56] }\end{array}$ & $62 / 52$ & CBT-MBSR & 8 weeks & Non-MBSR & $\begin{array}{c}\text { After } \\
\text { Treatment }\end{array}$ & $\begin{array}{l}\text { Mindfulness } \\
\text { Instructor }\end{array}$ & Not Reported \\
\hline
\end{tabular}


Table 1. Cont

\begin{tabular}{|c|c|c|c|c|c|c|c|c|c|}
\hline Variables & Tools & Study & $\begin{array}{c}n \\
\text { (Treatment/Control) }\end{array}$ & $\begin{array}{c}\text { Intervention } \\
\text { Type }\end{array}$ & $\begin{array}{l}\text { Intervention } \\
\text { Duration }\end{array}$ & $\begin{array}{l}\text { Control } \\
\text { Condition }\end{array}$ & $\begin{array}{c}\text { Inclusion } \\
\text { Criteria }\end{array}$ & Provider & $\begin{array}{l}\text { Stage of } \\
\text { Cancer }\end{array}$ \\
\hline \multirow{13}{*}{$\begin{array}{l}\text { Quality of } \\
\text { Life }\end{array}$} & EORTC-C30 & Bordeleau et al. [62] & $145 / 70$ & $\begin{array}{c}\text { SET + } \\
\text { Educational } \\
\text { Training }\end{array}$ & 12 weeks & $\begin{array}{c}\text { Educational } \\
\text { and } \\
\text { Relaxation } \\
\text { Training } \\
\end{array}$ & $\begin{array}{c}\text { After } \\
\text { Treatment }\end{array}$ & $\begin{array}{c}\text { Psychiatrist, } \\
\text { Psychologist, Social } \\
\text { Workers, Nurse }\end{array}$ & IV \\
\hline & EORTC-C30 & Chujo et al. [33] & $22 / 11$ & PET & 6 weeks & Wait List & $\begin{array}{c}\text { After } \\
\text { Treatment }\end{array}$ & $\begin{array}{l}\text { Psychiatrist and } \\
\text { Nurse }\end{array}$ & Not Reported \\
\hline & ABS & Antoni et al. [25] & $74 / 85$ & CBT & 10 weeks & $\begin{array}{l}\text { Educational } \\
\text { Program }\end{array}$ & $\begin{array}{c}\text { After } \\
\text { Treatment }\end{array}$ & Psychologist & From 0 to III \\
\hline & SF-12 & Fillion et al. [34] & $44 / 43$ & PET & 4 weeks & $\begin{array}{c}\text { General } \\
\text { Information }\end{array}$ & $\begin{array}{c}\text { After } \\
\text { Treatment }\end{array}$ & Nurses & From 0 to III \\
\hline & EORTC-C30 & Dolbeault et al. [32] & $81 / 87$ & PET & 8 weeks & Wait List & $\begin{array}{c}\text { After } \\
\text { Treatment }\end{array}$ & Two Therapists & I; II; III \\
\hline & EORTC-C30 & McKiernan et al. [63] & $23 / 30$ & $\begin{array}{c}\text { CBT }+ \\
\text { Educational } \\
\text { Training }\end{array}$ & 6 weeks & $\begin{array}{l}\text { Educational } \\
\text { Training }\end{array}$ & $\begin{array}{c}\text { After } \\
\text { Treatment }\end{array}$ & $\begin{array}{c}\text { Clinical } \\
\text { Psychologist and } \\
\text { Counselling } \\
\text { Psychologist } \\
\end{array}$ & $\begin{array}{c}\text { I; II; } \\
\text { Metastasis } \\
\text { and Lymph }\end{array}$ \\
\hline & $\begin{array}{l}\text { QoL Scales for } \\
\text { Korean } \\
\text { Cancer } \\
\text { Patients }\end{array}$ & Lee et al. [64] & $35 / 36$ & CBT & 6 weeks & Not Reported & $\begin{array}{c}\text { After } \\
\text { Treatment }\end{array}$ & Nurse & I; II; III \\
\hline & EORTC-C30 & Bjorneklett et al. [65] & $176 / 164$ & PET & $\begin{array}{l}1 \text { week }+4 \\
\text { meetings after } \\
2 \text { months }\end{array}$ & Wait List & $\begin{array}{c}\text { After } \\
\text { Treatment }\end{array}$ & Not Reported & I; II; III \\
\hline & FACT & Gudenkauf et al. [66] & $40 / 49$ & $\begin{array}{c}\text { CBT + } \\
\text { Relaxation } \\
\text { Training }\end{array}$ & 5 weeks & $\begin{array}{l}\text { Relaxation } \\
\text { Training }\end{array}$ & $\begin{array}{c}\text { After } \\
\text { Treatment }\end{array}$ & $\begin{array}{c}\text { Clinical Psychology } \\
\text { student }\end{array}$ & From 0 to III \\
\hline & EORTC-C30 & Jang et al. [51] & $12 / 11$ & CBT-MBSR & 12 weeks & Wait List & $\begin{array}{c}\text { After } \\
\text { Treatment }\end{array}$ & $\begin{array}{l}\text { Mental Health } \\
\text { Specialist }\end{array}$ & From 0 to III \\
\hline & WHO-5 & Johannsen et al. [52] & $46 / 61$ & CBT-MBSR & 8 weeks & Wait List & $\begin{array}{c}\text { After } \\
\text { Treatment }\end{array}$ & $\begin{array}{l}\text { Mindfulness } \\
\text { Instructor }\end{array}$ & Not Reported \\
\hline & SF-36 & Lengacher et al. [53] & $152 / 147$ & CBT-MBSR & 6 weeks & Usual Care & $\begin{array}{c}\text { After } \\
\text { Treatment }\end{array}$ & $\begin{array}{c}\text { Clinical } \\
\text { Psychologist }\end{array}$ & From 0 to III \\
\hline & SF-36 & Reich et al. [54] & $152 / 145$ & CBT-MBSR & 6 weeks & Usual Care & $\begin{array}{c}\text { After } \\
\text { Treatment }\end{array}$ & $\begin{array}{c}\text { Clinical } \\
\text { Psychologist }\end{array}$ & From 0 to III \\
\hline
\end{tabular}


Table 1. Cont.

\begin{tabular}{|c|c|c|c|c|c|c|c|c|c|}
\hline Variables & Tools & Study & $\begin{array}{c}n \\
\text { (Treatment/Control) }\end{array}$ & $\begin{array}{c}\text { Intervention } \\
\text { Type }\end{array}$ & $\begin{array}{c}\text { Intervention } \\
\text { Duration }\end{array}$ & $\begin{array}{l}\text { Control } \\
\text { Condition }\end{array}$ & $\begin{array}{c}\text { Inclusion } \\
\text { Criteria }\end{array}$ & Provider & $\begin{array}{c}\text { Stage of } \\
\text { Cancer }\end{array}$ \\
\hline \multirow{10}{*}{ Mood } & ABS & Kissane et al. [24] & $145 / 141$ & $\begin{array}{c}\text { CBT + } \\
\text { Relaxation } \\
\text { Training }\end{array}$ & 20 weeks & $\begin{array}{l}\text { Relaxation } \\
\text { Training }\end{array}$ & $\begin{array}{c}\text { Inpatient } \\
\text { Treatment }\end{array}$ & Two Therapists & I; II \\
\hline & POMS & Chujo et al. [33] & $22 / 11$ & PET & 6 weeks & Wait List & $\begin{array}{c}\text { After } \\
\text { Treatment }\end{array}$ & $\begin{array}{l}\text { Psychiatrist and } \\
\text { Nurse }\end{array}$ & Not Reported \\
\hline & BSI & Cohen and Fried [67] & $38 / 37$ & $\begin{array}{c}\text { CBT + } \\
\text { Relaxation } \\
\text { Training }\end{array}$ & 9 weeks & Usual Care & $\begin{array}{c}\text { After } \\
\text { Treatment }\end{array}$ & $\begin{array}{l}\text { Cohen (Expert in } \\
\text { Psycho-Oncology) }\end{array}$ & I; II \\
\hline & IES & Kissane et al. [57] & $115 / 56$ & SET & 13 weeks & $\begin{array}{l}\text { Relaxation } \\
\text { Training }\end{array}$ & $\begin{array}{c}\text { After } \\
\text { Treatment }\end{array}$ & Therapist & IV \\
\hline & POMS & Classen et al. [27] & $151 / 148$ & SET & 12 weeks & $\begin{array}{l}\text { Educational } \\
\text { Program }\end{array}$ & $\begin{array}{c}\text { After } \\
\text { Treatment }\end{array}$ & $\begin{array}{l}\text { Nurse, Psychologist, } \\
\text { Social Workers }\end{array}$ & From 0 to III \\
\hline & POMS & Fillion et al. [34] & $44 / 43$ & PET & 4 weeks & $\begin{array}{c}\text { General } \\
\text { Information }\end{array}$ & $\begin{array}{c}\text { After } \\
\text { Treatment }\end{array}$ & Nurses & From 0 to III \\
\hline & POMS & Dolbeault et al. [32] & $81 / 87$ & PET & 8 weeks & Wait List & $\begin{array}{c}\text { After } \\
\text { Treatment }\end{array}$ & Two Therapists & I; II; III \\
\hline & POMS & McKiernan et al. [63] & $23 / 30$ & $\begin{array}{c}\text { CBT }+ \\
\text { Educational } \\
\text { Training }\end{array}$ & 6 weeks & $\begin{array}{l}\text { Educational } \\
\text { Training }\end{array}$ & $\begin{array}{c}\text { After } \\
\text { Treatment }\end{array}$ & $\begin{array}{c}\text { Clinical } \\
\text { Psychologist and } \\
\text { Counselling } \\
\text { Psychologist }\end{array}$ & $\begin{array}{c}\text { I; II; } \\
\text { Metastasis } \\
\text { and Lymph }\end{array}$ \\
\hline & POMS & Lechner et al. [59] & $57 / 57$ & CBT-CBSM & 10 weeks & $\begin{array}{c}\text { Educational } \\
\text { Program }\end{array}$ & $\begin{array}{c}\text { After } \\
\text { Treatment }\end{array}$ & $\begin{array}{c}\text { Clinical } \\
\text { Psychologist }\end{array}$ & From 0 to IV \\
\hline & ABS & Gudenkauf et al. [66] & $40 / 49$ & $\begin{array}{c}\text { CBT }+ \\
\text { Relaxation } \\
\text { Training }\end{array}$ & 5 weeks & $\begin{array}{l}\text { Relaxation } \\
\text { Training }\end{array}$ & $\begin{array}{c}\text { After } \\
\text { Treatment }\end{array}$ & $\begin{array}{c}\text { Clinical Psychology } \\
\text { student }\end{array}$ & From 0 to III \\
\hline
\end{tabular}

ABS: Affect Balance Scale; BDI: Beck Depression Inventory; BSI: Brief Symptom Inventory; CBT-CBSM: Cognitive Behavioural Therapy-Cognitive Behavioural Stress Management;

CBT-MBSR: Cognitive Behavioural Therapy-Mindfulness-Based Stress Reduction Program; CBT-MAP: Cognitive Behavioural Therapy-Mindful Awareness Practices; CBT-CDCT:

Cognitively-Based Compassion Training; CES-D: Centre for Epidemiologic Studies Depression Scale; EORTC-C30: European Organisation for Research and the Treatment of Cancer Core

Quality of Life Questionnaire; FACT: Functional Assessment of Cancer Therapy; HADS: Hospital Anxiety and Depression Scale; MCS-12: Medical Outcomes Studies Short-Form General

Health Survey; PAI: Personality Assessment Inventory; SF-12/SF-36: Short-Form Health Survey; PET: psycho-educational therapy; POMS: Profile of Mood States; SET: supportive-expressive

group therapy; STAI: State-Trait Anxiety Inventory; WHO-5: World Health Organisation-5 Well-Being Index; IES: Impact of Events Scale. 


\section{Results}

\subsection{Study Selection}

The flow diagram of the screened studies is presented in Figure 1. Four hundred and forty-nine studies were retrieved from databases $(n=438)$ and other sources $(n=11)$. After excluding duplicates $(n=105)$ and studies not matching inclusion criteria $(n=233), 111$ full texts were considered for the systematic review. After reading the full-texts, 25 studies from January 2003 to April 2017 met all inclusion criteria and were included in the meta-analysis. Some selected studies analysed the effect of the intervention on different psychological aspects providing distinct outcomes. According to the aims of this study (i.e., to analyse the effectiveness of some psychological interventions in women with breast cancer considering specific psychological aspects such as mood, depression, anxiety and quality of life), studies with multiple outcomes were considered as independent. For these reasons, the meta-analysis included 45 independent studies (see Figure 1 and Table 1).

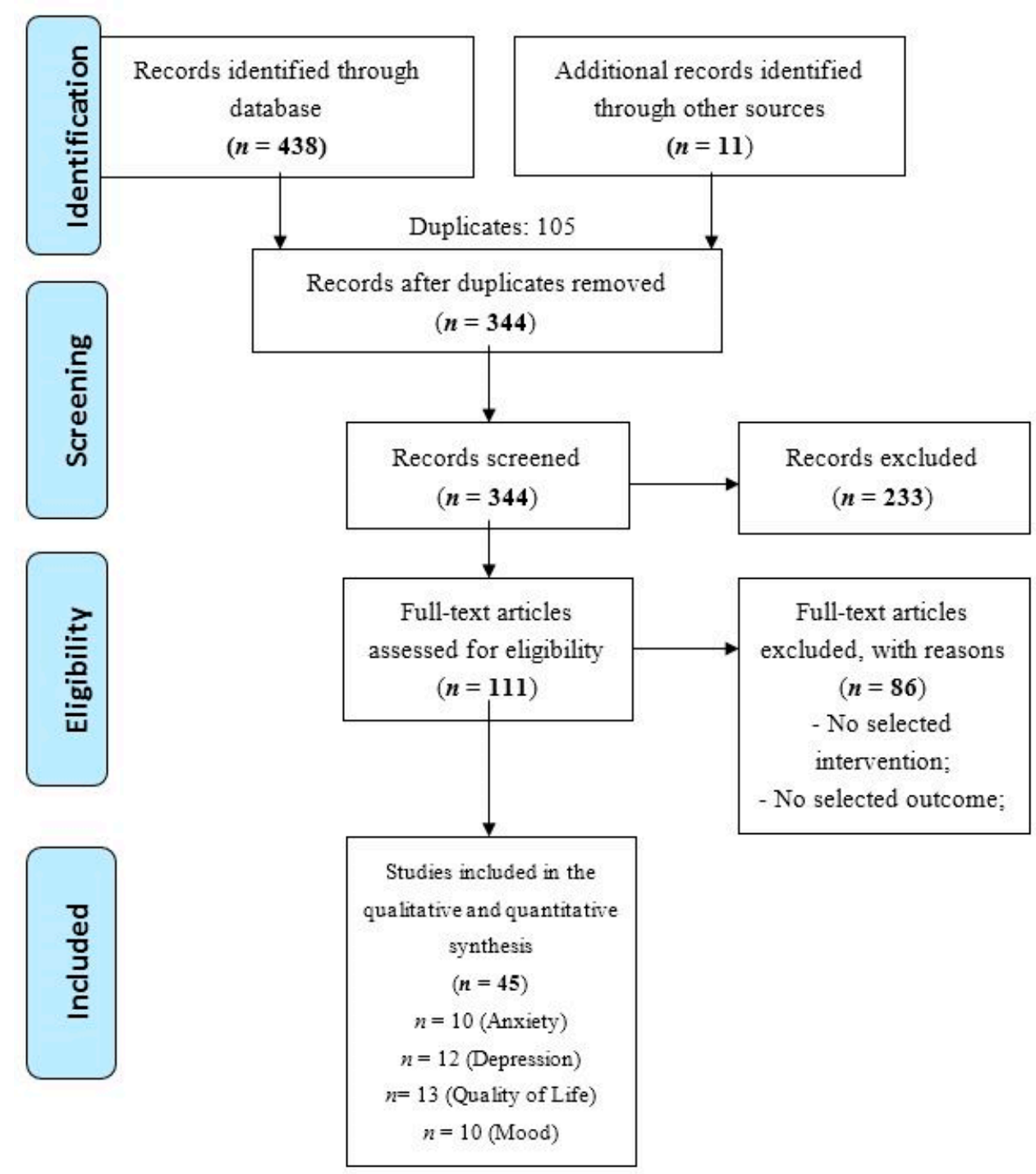

Figure 1. The study screening process. 


\subsection{Characteristics of Included Studies}

The characteristics of selected studies are presented in Table 1.

The total number of participants with cancer included in the clinical trials was 8472 , considering the pre-intervention evaluation, and 7834 viewing the post-treatment assessment, with a drop-out of around $8 \%(n=638)$.

Ten studies considered the effect of treatment on anxiety [24,30,32,50-56]; twelve studies considered the effect of treatment on depression [24,30,50-61]; ten studies considered the effect of the treatment on mood $[24,28,32-34,57,59,63,66,67]$; thirteen studies considered the effects of the treatment on the quality of life $[25,32-34,51-54,59,62-66]$.

Seventeen studies used cognitive behavioural therapy (CBT), four studies used psycho-educational treatment (PET), and four studies analysed supportive-expressive group therapy (SET) (see Tables 1 and 2).

The providers who implemented the therapies were mainly psychologists, nurses, psychologists and psychiatrists together, as well as mindfulness experts (see Table 1). In some circumstances, the providers were a multidisciplinary team or postgraduate students, while three studies did not give specific information about the providers $[58,61,65]$.

The duration of the interventions ranged from one [65] to twenty weeks [24] (see Table 1).

Several questionnaires were used to measure the psychological variables. To evaluate anxiety, the following questionnaires were used: the State-Trait Anxiety Inventory (STAI) [68], the Hospital Anxiety and Depression Scale (HADS) [69] and the Personality Assessment Inventory (PAI) [70] (see Table 1). For the assessment of depression, the Beck Depression Inventory (BDI) [71], the Centre for Epidemiologic Studies Depression Scale (CES-D) [72], the Functional Assessment of Cancer Therapy (FACT) [73], the PAI [70] and the HADS [69] were employed (see Table 1). Mood was assessed by using the Affect Balance Scale (ABS) [74], the Brief Symptom Inventory (BSI) [75] and the Profile of Mood States (POMS) [76] (see Table 1). For the assessment of quality of life, the following questionnaires were used: the ABS [74], the European Organisation for Research and the Treatment of Cancer Core Quality of Life Questionnaire (EORTC-C30) [77], the FACT [73], the Medical Outcomes Studies Short-Form General Health Survey (MCS-12) [78], the Quality of Life Scales for Korean Patients with Cancer [79], the World Health Organisation-5 Well-Being Index (WHO-5) [80] (see Table 1). 
Table 2. Risk of bias assessment for each study selected for the meta-analysis.

\begin{tabular}{|c|c|c|c|c|c|c|c|c|}
\hline Study & $\begin{array}{l}\text { Intervention } \\
\text { Type }\end{array}$ & $\begin{array}{c}\text { Random } \\
\text { Sequence } \\
\text { Generation }\end{array}$ & $\begin{array}{l}\text { Allocation } \\
\text { Concealment }\end{array}$ & $\begin{array}{c}\text { Blinding } \\
\text { (Outcome } \\
\text { Assessment) }\end{array}$ & $\begin{array}{l}\text { Incomplete } \\
\text { Outcome }\end{array}$ & $\begin{array}{l}\text { Selective } \\
\text { Reporting }\end{array}$ & $\begin{array}{c}\text { Other } \\
\text { Sources of } \\
\text { Bias }\end{array}$ & $\begin{array}{c}\text { Overall } \\
\text { Risk of Bias }\end{array}$ \\
\hline Bordeleau et al. [62] & SET & + & $?$ & $?$ & $?$ & + & $?$ & $?$ \\
\hline Kissane et al. [24] & CBT & + & + & + & $?$ & $?$ & $?$ & $?$ \\
\hline Chujo et al. [33] & PET & - & - & $?$ & - & + & $?$ & - \\
\hline Antoni et al. [23] & CBT & $?$ & $?$ & + & + & $?$ & $?$ & $?$ \\
\hline Cohen and Fried [67] & CBT & $?$ & $?$ & + & + & + & $?$ & $?$ \\
\hline Kissane et al. [57] & SET & + & $?$ & - & $?$ & $?$ & $?$ & - \\
\hline Classen et al. [27] & SET & + & $?$ & $?$ & $?$ & - & $?$ & - \\
\hline Fillion et al. [34] & PET & $?$ & $?$ & $?$ & + & + & $?$ & $?$ \\
\hline Dolbeault et al. [32] & PET & + & + & $?$ & $?$ & + & $?$ & $?$ \\
\hline McKiernan et al. [63] & CBT & - & - & $?$ & $?$ & $?$ & $?$ & - \\
\hline Lee et al. [64] & CBT & - & - & - & $?$ & $?$ & $?$ & - \\
\hline Björneklett et al. [65] & PET & + & + & + & $?$ & + & $?$ & $?$ \\
\hline Groarke et al. [50] & CBT & + & + & $?$ & + & + & $?$ & $?$ \\
\hline Bower et al. [58] & CBT & $?$ & $?$ & $?$ & + & + & $?$ & $?$ \\
\hline Lechner et al. [59] & CBT & $?$ & $?$ & $?$ & + & + & $?$ & $?$ \\
\hline Dodds et al. [60] & CBT & + & $?$ & $?$ & $?$ & + & $?$ & $?$ \\
\hline Gudenkauf et al. [66] & CBT & + & + & + & $?$ & + & $?$ & $?$ \\
\hline Ho et al. [30] & SET & + & $?$ & $?$ & + & + & $?$ & $?$ \\
\hline Jang et al. [51] & CBT & $?$ & $?$ & $?$ & + & + & $?$ & $?$ \\
\hline Johannsen et al. [52] & CBT & + & $?$ & $?$ & + & + & $?$ & $?$ \\
\hline Langacher et al. [53] & CBT & + & $?$ & $?$ & + & + & $?$ & $?$ \\
\hline Reich et al. [54] & CBT & $?$ & $?$ & - & + & $?$ & $?$ & - \\
\hline Zhang et al. [55] & CBT & $?$ & - & $?$ & + & + & $?$ & - \\
\hline Boyle et al. [61] & CBT & - & $?$ & $?$ & + & $?$ & $?$ & - \\
\hline Kenne Sarenmalm et al. [56] & CBT & + & + & $?$ & + & + & $?$ & $?$ \\
\hline
\end{tabular}

$(+)=$ low risk of bias; $(-)=$ high risk of bias; $(?)=$ unclear risk of bias. 


\subsection{Qualitative Assessment of the Risk of Bias}

The risk of bias was assessed. Overall, about one-third of the articles $(n=8 / 25 ; 32 \%)$ showed a high risk of bias on at least one criterion (Table 2). The remaining studies $(n=17 / 25 ; 68 \%$ ) presented at least some concerns (e.g., allocation concealment, incomplete outcome, outcome assessment). The highest risks of bias were identified in both the randomisation process and the allocation of participants $(n$ $=4 / 25)$, while the lowest risk of bias was identified in the selective reports of the data $(n=18 / 28)$ (Figure 2).

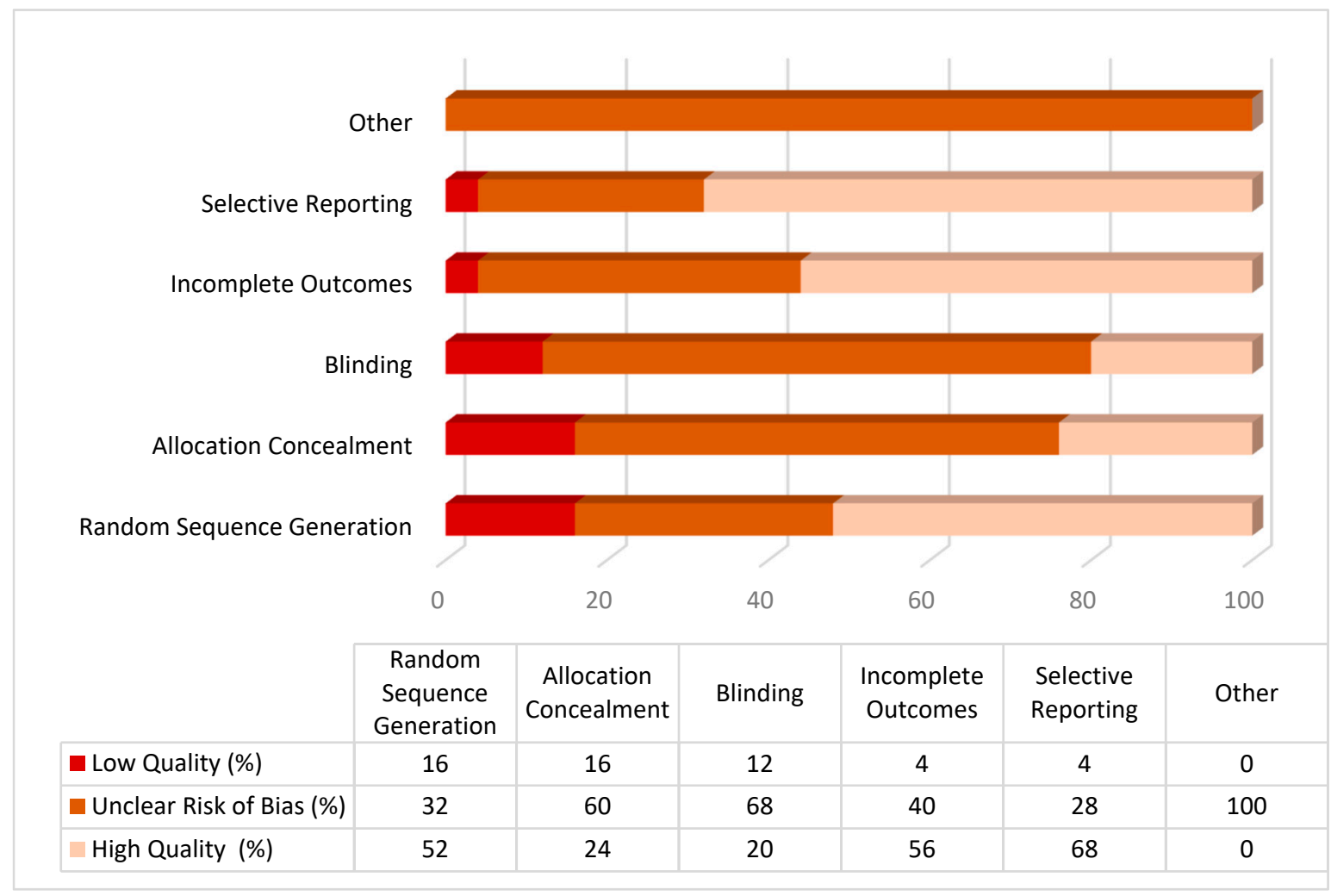

Figure 2. Qualitative assessment (\% of studies for each condition of risk of bias).

\subsection{Overall Effect Size Considering Differences between Intervention and Control Group after the Treatment}

Overall effect sizes were reported considering both different psychological variables and interventions.

\subsection{Anxiety}

The overall ES of the studies about anxiety reduction on women with breast cancer was medium $(0.39 ; 95 \% \mathrm{CI} ;-0.91$ to 0.14$)$ with a high heterogeneity among the studies $\left(p<0.0001 ; \mathrm{I}^{2}=98 \%\right)$ (see Figure 3).

The ES ranged between 0.10 [30] and -2.59 [51].

The study of Jang et al. [51] showed the most significant effect size (-2.59); four studies reported medium ESs, ranging between -0.40 [55] and -0.45 [32]. The other studies reported small ESs, ranging between -0.09 [54] and -0.21 [24].

Considering subgroups analyses, significant differences among studies were observed about types of intervention $(p=0.001)$ and types of the provider $(p=0.0001)$. Specifically, studies on CBT and PET interventions showed larger ESs (see Table 3), and larger ESs were observed on studies that included different types of providers (ES $=-0.94)$. No differences were shown considering inclusion criteria of intervention group $(p=0.38)$, duration of intervention $(p=0.52)$ or control group condition $(p=0.10)$. 
Table 3. Effect size (Cohen's d) for each variable.

\begin{tabular}{|c|c|c|c|}
\hline Analyses & $\begin{array}{c}\text { Number of } \\
\text { Studies }\end{array}$ & Effect Size $(95 \%$ CI) & $\begin{array}{l}\text { Heterogeneity } \\
\left(\mathrm{I}^{2 \%} \%\right)\end{array}$ \\
\hline \multicolumn{4}{|l|}{ Anxiety } \\
\hline Overall Effect Size & 10 & $-0.39[-0.91,0.14]$ & 98 \\
\hline Type of Intervention & & & - \\
\hline Cognitive Behavioural Therapy & 8 & $-0.45[-1.14,0.24]$ & 97 \\
\hline Psycho-Educational Therapy & 1 & $-0.45[-0.49,-0.40]$ & - \\
\hline Supportive-Expressive Therapy & 1 & $0.10[0.02,0.18]$ & \\
\hline \multicolumn{4}{|l|}{ Type of Provider } \\
\hline Psychologist & 6 & $-0.14[-0.31,0.02]$ & 95 \\
\hline Psychologist + Psychiatrist & 1 & $-0.45[-0.49,-0.40]$ & - \\
\hline Nurse & - & - & - \\
\hline Other & 3 & $-0.94[-4.47,2.58]$ & 98 \\
\hline \multicolumn{4}{|l|}{ Not Reported } \\
\hline \multicolumn{4}{|l|}{ Duration of Intervention } \\
\hline Less than 6 Weeks & 1 & $-0.19[-0.22,-0.15]$ & - \\
\hline Between 6 and 12 Weeks & 8 & $-0.45[-1.15,0.26]$ & 98 \\
\hline More than 12 Weeks & - & - & - \\
\hline Not Reported & 1 & $-0.20[-0.23,-0.18]$ & - \\
\hline \multicolumn{4}{|l|}{ Control Group } \\
\hline Alternative Intervention & 3 & $0.003[-0.46,0.47]$ & 98 \\
\hline Wait List & 3 & $-1.12[-4.16,1.90]$ & 93 \\
\hline Standard Care & 4 & $-0.18[-0.40,0.04]$ & 93 \\
\hline \multicolumn{4}{|l|}{ Not Reported } \\
\hline \multicolumn{4}{|l|}{ Inclusion Criteria } \\
\hline In Patient & 1 & $-0.21[-0.24,-0.18]$ & \\
\hline After Treatment & 9 & $-0.41[-1.01,0.19]$ & 98 \\
\hline Not Reported & - & - & - \\
\hline \multicolumn{4}{|l|}{ Depression } \\
\hline Overall Effect Size & 12 & $-0.35[-0.79,-0.10]$ & 98 \\
\hline \multicolumn{4}{|l|}{ Type of Intervention } \\
\hline Cognitive Behavioural Therapy & 10 & $-0.42[-0.96,0.12]$ & 98 \\
\hline Psycho-Educational Therapy & - & - & - \\
\hline Supportive-Expressive Therapy & 2 & $-0.10[-2.18,2.16]$ & 98 \\
\hline \multicolumn{4}{|l|}{ Type of Provider } \\
\hline Psychologist & 7 & $-0.15[-0.37,0.06]$ & 97 \\
\hline Psychologist + Psychiatrist & - & - & - \\
\hline Nurse & - & - & - \\
\hline Other & 3 & $-1.04[-3.85,1.76]$ & 97 \\
\hline Not Reported & 2 & $-0.08[-9.44,9.26]$ & 99 \\
\hline \multicolumn{4}{|l|}{ Duration of Intervention } \\
\hline Less than 6 Weeks & 1 & $-0.08[-0.11,-0.04]$ & - \\
\hline Between 6 and 12 Weeks & 8 & $-0.40[-1.12,0.33]$ & 98 \\
\hline More than 12 Weeks & 1 & $-0.18[-0.21,-0.14]$ & - \\
\hline Not Reported & 2 & $-0.39[-4.31,3.51]$ & 93 \\
\hline \multicolumn{4}{|l|}{ Control Group } \\
\hline Alternative Intervention & 5 & $-0.18[-0.53,0.17]$ & 98 \\
\hline Wait List & 4 & $-0.79[-2.72,1.14]$ & 99 \\
\hline Standard Care & 3 & $-0.11[-0.16,-0.05]$ & 50 \\
\hline Not Reported & - & - & - \\
\hline \multicolumn{4}{|l|}{ Inclusion Criteria } \\
\hline In Patient & 1 & $-0.10[-0.13,-0.08]$ & \\
\hline After Treatment & 11 & $-0.37[-0.86,0.12]$ & 98 \\
\hline Not Reported & - & - & - \\
\hline
\end{tabular}


Table 3. Cont.

\begin{tabular}{|c|c|c|c|}
\hline Analyses & $\begin{array}{c}\text { Number of } \\
\text { Studies }\end{array}$ & Effect Size (95\% CI) & $\begin{array}{c}\text { Heterogeneity } \\
\left(\mathrm{I}^{\mathbf{2} \%)}\right.\end{array}$ \\
\hline \multicolumn{4}{|l|}{ Mood } \\
\hline Overall Effect Size & 10 & $-0.17[-0.41,0.06]$ & 98 \\
\hline \multicolumn{4}{|l|}{ Type of Intervention } \\
\hline Cognitive Behavioural Therapy & 5 & $-0.12[-0.64,0.39]$ & 98 \\
\hline Psycho-Educational Therapy & 3 & $-0.34[-1.05,0.36]$ & 97 \\
\hline Supportive-Expressive Therapy & 2 & $-0.07[-1.70,1.56]$ & 99 \\
\hline \multicolumn{4}{|l|}{ Type of Provider } \\
\hline Psychologist & 6 & $-0.12[-0.51,0.25]$ & 99 \\
\hline Psychologist + Psychiatrist & - & - & - \\
\hline Nurse & 1 & $-0.02[-0.11,0.06]$ & - \\
\hline Other & 3 & $-0.33[-1.19,0.54]$ & 98 \\
\hline \multicolumn{4}{|l|}{ Not Reported } \\
\hline \multicolumn{4}{|l|}{ Duration of Intervention } \\
\hline Less than 6 weeks & 2 & $0.001[-0.36,0.37]$ & 0 \\
\hline Between 6 and 12 Weeks & 5 & $-0.31[-0.87,0.24]$ & 96 \\
\hline More than 12 Weeks & 2 & $-0.07[-1.70,1.56]$ & 99 \\
\hline Not Reported & 1 & $.0 .08[-0.10,-0.05]$ & 99 \\
\hline \multicolumn{4}{|l|}{ Control Group } \\
\hline Alternative Intervention & 6 & $-0.03[-0.35,0.29]$ & 98 \\
\hline Wait List & 3 & $-0.34[-1.06,0.36]$ & 96 \\
\hline Standard Care & 1 & $-0.57[-0.68,-0.46]$ & - \\
\hline \multirow{2}{*}{\multicolumn{4}{|c|}{$\begin{array}{l}\text { Not Reported } \\
\text { Inclusion Criteria }\end{array}$}} \\
\hline & & & \\
\hline In Patient & 1 & $-0.08[-0.10,0.05]$ & - \\
\hline After Treatment & 9 & $-0.18[-0.46,0.08]$ & 98 \\
\hline Not Reported & - & - & - \\
\hline \multicolumn{4}{|l|}{ Quality of Life } \\
\hline Overall Effect Size & 13 & $0.38[-0.07,0.84]$ & 98 \\
\hline \multicolumn{4}{|l|}{ Type of Intervention } \\
\hline Cognitive Behavioural Therapy & 8 & $0.55[-0.25,1.35]$ & 99 \\
\hline Psycho-Educational Therapy & 4 & $0.17[0.02,0.32]$ & 89 \\
\hline Supportive-Expressive Therapy & 1 & $-0.11[-0.15,-0.07]$ & - \\
\hline \multicolumn{4}{|l|}{ Type of Provider } \\
\hline Psychologist & 6 & $0.08[-0.18,0.34]$ & 93 \\
\hline Psychologist + Psychiatrist & 2 & $-0.03[-1.17,1.12]$ & 96 \\
\hline Nurse & 3 & $0.72[-1.18,2.63]$ & 99 \\
\hline Other & 1 & $2.56[1.93,3.18]$ & - \\
\hline Not Reported & 1 & $0.20[0.17,0.22]$ & - \\
\hline \multicolumn{4}{|l|}{ Duration of Intervention } \\
\hline Less than 6 Weeks & 2 & $0.11[-1.34,1.57]$ & 97 \\
\hline Between 6 and 12 Weeks & 10 & $0.46[-0.15,1.08]$ & 90 \\
\hline More than 12 Weeks & - & - & - \\
\hline Not Reported & 1 & $0.20[0.17,0.22]$ & - \\
\hline \multicolumn{4}{|l|}{ Control Group } \\
\hline Alternative Intervention & 6 & $0.27[-0.41,0.96]$ & 98 \\
\hline Wait List & 5 & $0.71[-0.53,1.96]$ & 97 \\
\hline Standard Care & 2 & $-0.03[-0.18,0.12]$ & 36 \\
\hline Not Reported & - & - & - \\
\hline \multicolumn{4}{|l|}{ Inclusion Criteria } \\
\hline In Patient & - & - & - \\
\hline After treatment & 13 & $0.38[-0.07,0.84]$ & 98 \\
\hline Not Reported & - & - & - \\
\hline
\end{tabular}




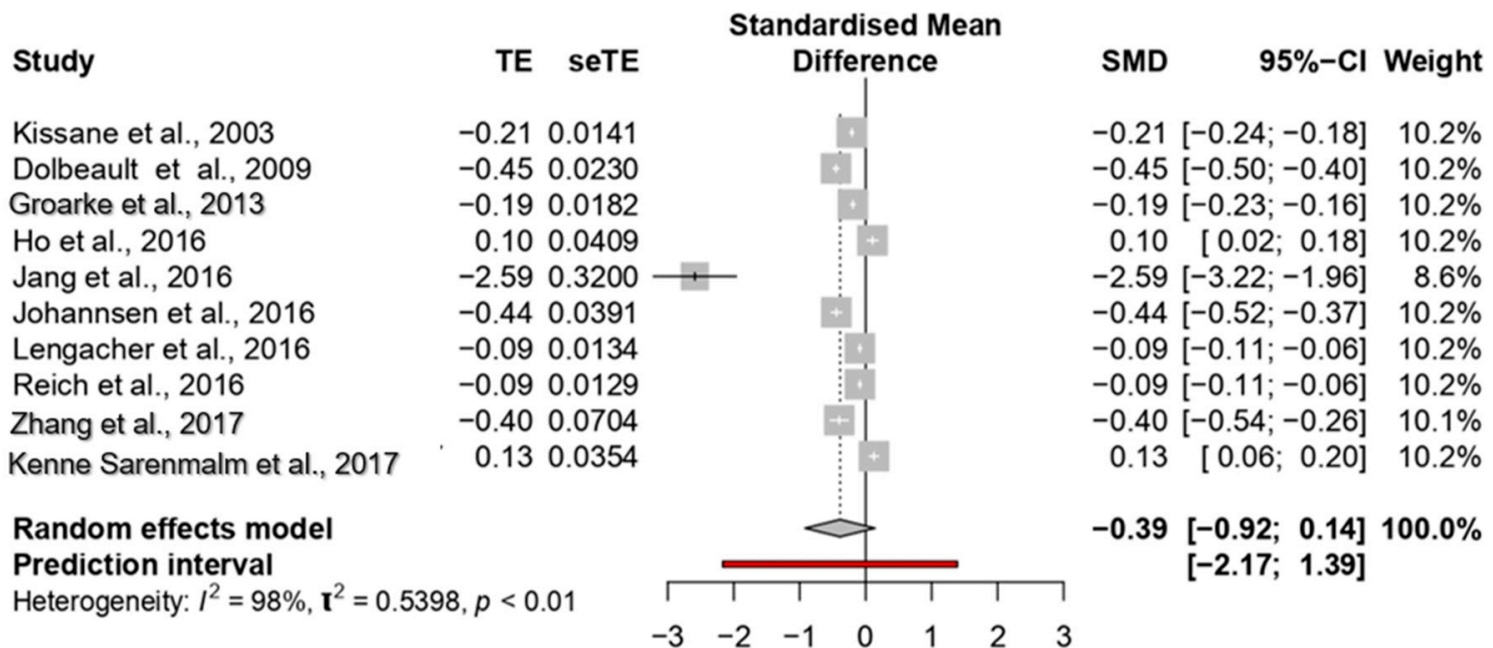

Figure 3. Forest plot of the studies considering psychological interventions focused on reducing anxiety.

\subsection{Depression}

The overall ES of psychological interventions on depression was medium $(-0.35 ; 95 \%$ CI: -0.79 to -0.10$)$ (Figure 4$)$. The meta-analysis showed higher heterogeneity among the studies $(p<0.0001$; $I^{2}=98 \%$ ) (see Figure 4$)$.

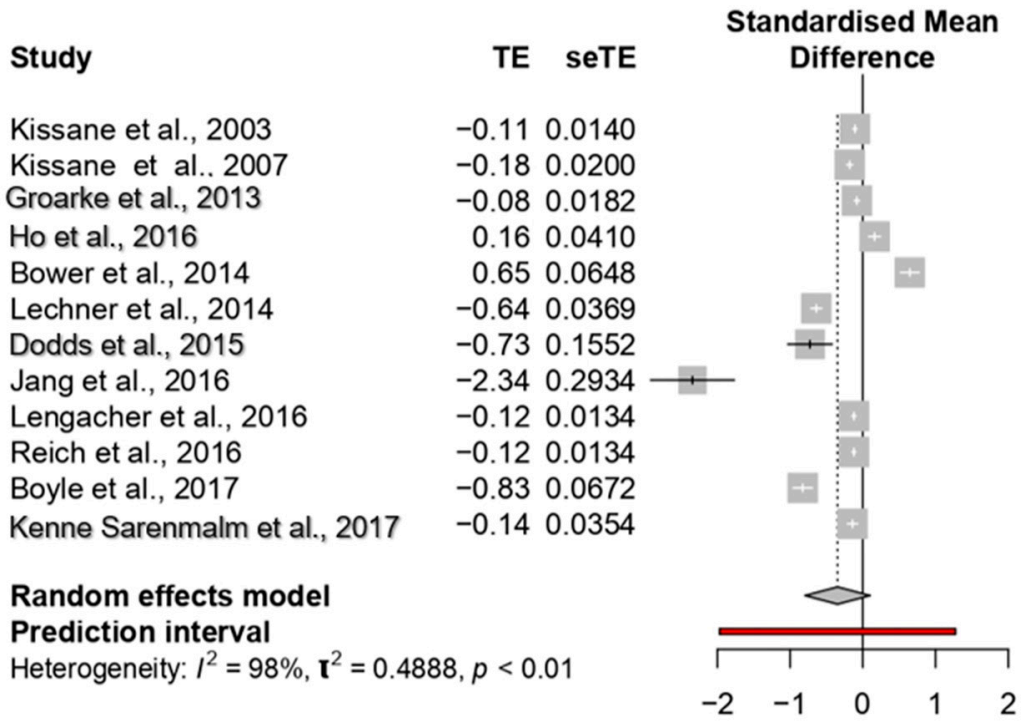

\section{SMD $\quad 95 \%-\mathrm{Cl}$ Weight}

$-0.11[-0.14 ;-0.08] \quad 8.5 \%$

$-0.18[-0.22 ;-0.14] \quad 8.5 \%$

$-0.08[-0.12 ;-0.05] \quad 8.5 \%$

$0.16[0.08 ; 0.24] \quad 8.5 \%$

$0.65 \quad[0.52 ; 0.77] \quad 8.4 \%$

$-0.64[-0.71 ;-0.57] \quad 8.5 \%$

$-0.73[-1.03 ;-0.42] \quad 8.1 \%$

$-2.34[-2.92 ;-1.77] \quad 7.2 \%$

$-0.12[-0.15 ;-0.10] \quad 8.5 \%$

$-0.12[-0.15 ;-0.10] \quad 8.5 \%$

$-0.83[-0.96 ;-0.69] \quad 8.4 \%$

$-0.14[-0.21 ;-0.07] \quad 8.5 \%$

$-0.35[-0.80 ; 0.10] 100.0 \%$

$[-1.97 ; 1.28]$

Figure 4. Forest plot of the studies considering psychological interventions focused on reducing depression.

Two studies showed a large ES, ranging between -0.83 [61] and -2.34 [51]; four studies reported medium ESs, ranging between -0.64 [59] and -0.73 [60]. The other studies reported small ESs ranging between -0.08 [50] and -0.18 [57].

Regarding subgroup analyses, no differences among studies were observed comparing different types of intervention $(p=0.16)$, types of provider $(p=0.40)$ or control group condition $(p=0.45)$. However, there was a significant difference in the duration of interventions $(p=0.001)$. The largest ES was found in the interventions lasting from 6 to 12 weeks $(-0.40)$.

\subsection{Mood}

The overall ES in the improvement of mood was small $(-0.17 ; 95 \%$ CI: -0.41 to 0.06$)$. The meta-analysis showed high heterogeneity among the studies $\left(p<0.0001 ; \mathrm{I}^{2}=98 \%\right)$ (see Figure 5$)$. 


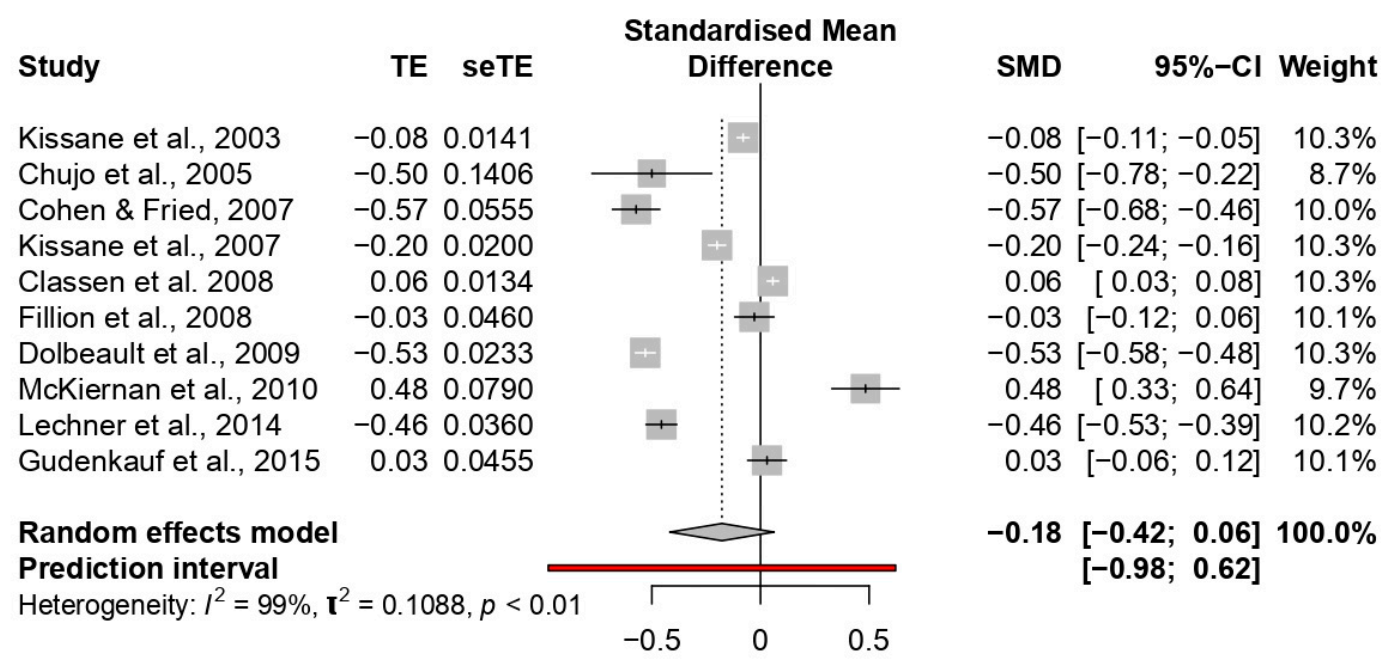

Figure 5. Forest plot of the studies considering psychological interventions focused on increasing mood.

No study showed a large ES; eight studies reported medium ESs ranging between - 0.20 [57] and -0.57 [67]. All the other studies reported small ESs ranging between -0.02 [34] and -0.08 [24].

Subgroup analyses of different treatments on mood did not show significant differences $(p=0.41)$. Moreover, no differences were found when the treatment group $(p=0.46)$ and intervention duration $(p=0.11)$ were considered. However, significant differences were observed when the type of provider $(p<0.01)$ and the control group condition $(p<0.0001)$ were considered. Specifically, higher ESs were observed in the studies with various types of the provider (-0.40; e.g., counsellor, postgraduate student, etc.), and that used standard care in the control group (-0.56) (see Table 3$)$.

\subsection{Quality of Life}

The overall ES for improved quality of life was medium (0.38; $95 \%$ CI: -0.07 to 0.84$)$. High heterogeneity among the studies $\left(p<0.0001 ; \mathrm{I}^{2}=98 \%\right)$ was found (see Figure 6 ).

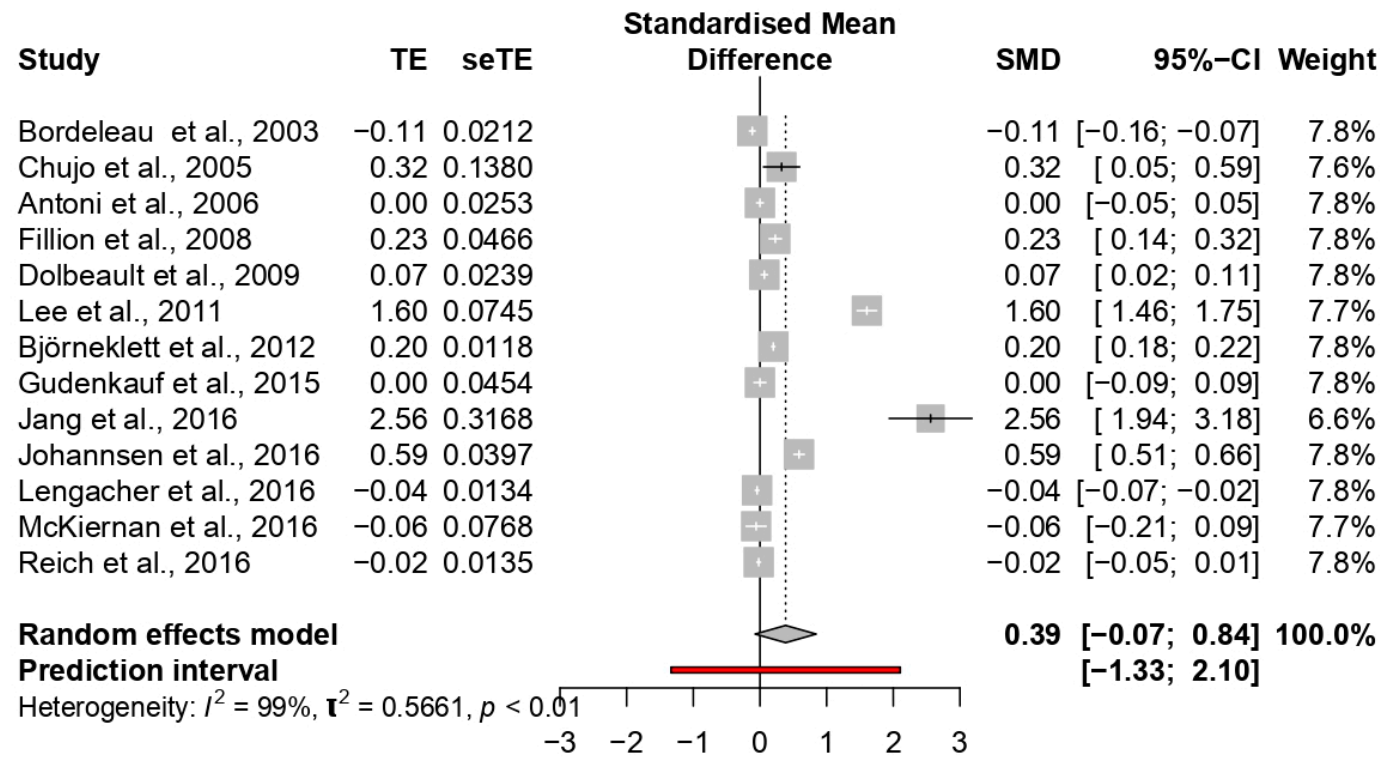

Figure 6. Forest plot of the studies considering psychological interventions focused on increasing quality of life. 
Two studies showed a large ES, ranging between 1.60 [64] and 2.56 [51]; four studies reported medium ESs ranging between 0.32 [33] and 0.59 [52]; the other studies reported small ESs ranging between 0 [25] and 0.20 [65].

In subgroup analyses, significant differences were observed considering types of treatment $(p=0.0001)$ and types of the provider $(p=0.0001)$. The highest ESs were highlighted in the studies considering CBT interventions ( $E S=0.55)$ and different types of providers $(E S=2.56)$. No differences emerged considering intervention duration $(p=0.30)$ or control group condition $(p=0.15)$.

\section{Discussion}

The goals of this study were to assess the clinical effectiveness of psychological treatments (psycho-educational intervention, cognitive behavioural therapy or supportive-expressive therapy) on different psychological outcomes (anxiety, depression, mood and quality of life) in women with a breast cancer diagnosis. Overall, the present review showed the low quality of the studies on this topic, characterised by few and heterogeneous methodological information, which influenced the meta-analysis results.

However, our results showed medium effect sizes that indicate moderate effectiveness of the selected approaches in improving the psychological well-being of patients with breast cancer, almost when the psychological variables analysed in this review are considered as the outcomes.

Several subgroup analyses about specific variables (e.g., approach, timing, provider) highlighted an influence of some characteristics of the sample and the methods adopted in the clinical trials, which could have determined the high heterogeneity among the studies as well as the different treatment efficacies.

Considering the effect of psychological interventions on patient anxiety levels, the overall effect size of the treatment $(-0.39)$ was similar to that reported by previous findings, considering the heterogeneous population of patients with cancer $(E S=-0.42)$ [80] or considering specifically breast cancer $(E S=0.40)$ [35]. Although other studies showed higher effect sizes of the intervention aiming to reduce anxiety in patients with breast cancer $[35,36,39,42,80,81]$, this difference could be related to the choice to analyse only group therapies, disregarding one-to-one interventions $[35,36,80,81]$. Individual treatments could be more effective in reducing anxiety and improving personal skills aiming to manage the distress related to the cancer condition.

Interestingly, when the outcome was anxiety, it was related to the differences in the efficacy of the approaches considered. The clinical efficacy of cognitive behavioural therapy was found to be comparable to the benefits of psycho-educational interventions. Conversely, supportive-expressive interventions appeared to fail in showing significant clinical benefits in the reduction of anxiety symptoms; these results agree with those observed by previous studies $[22,66,82,83]$. The effectiveness of cognitive behavioural therapy on anxiety is well known [82], and many studies [22,66,83] highlighted its effects on heterogeneous populations of patients with cancer. Additionally, the psycho-educational approach appears to increase personal medical-condition awareness and its related psycho-physical consequences, such as improved general psychological well-being and decreased anxiety [22,84]. The modification of maladaptive cognitive schemas and the improvement of affective and emotional states are of clinical importance; however, due to the heterogeneity of the studies, also in sub-groups analyses, we cannot generalise our results.

A moderate-to-strong clinical impact in patients with breast cancer appears to be led by different provider types, including various specialist figures (nurse, psychologist and psychiatrist together). We hypothesised that this effect might reflect higher perceived support due to the interaction with more specialists during the treatment.

For the depression outcome, a clinical impact of the treatment $(E S=-0.35)$ was observed. Depression represents an essential, potentially fatal, but treatable complication of breast cancer in women. Depression affects the quality of life of patients and caregivers, and it contributes to lower adherence to medical treatment [85]. Identifying an intervention which allows reducing depressive 
symptoms related to breast cancer is relevant to improving the quality of life of patients during treatment and in the follow-up phase.

CBT appears to be the best-known treatment for depression [86] because it can help to modify maladaptive information processes associated with negative thinking, which has a causal role in the maintenance of depression. This treatment has superior efficacy to other psychotherapies in the acute treatment of depression and the reduction of depressive symptomatology. Our results also confirmed the effectiveness of CBT in reducing depressive symptoms in patients with breast cancer, proving that the tools furnished by this intervention are more helpful than other treatments (e.g., supportive-expressive group therapy) in counteracting depression $[87,88]$. These findings highlight the importance of providing CBT to women with breast cancer, and could help to increase adherence to medical treatment.

Considering mood, the meta-analysis showed an overall low effect size $(E S=-0.20)$, although the psycho-educational approach appears to be partially better than the other types of interventions in increasing the mood of the patients $(E S=-0.37$ ). Its efficacy could be related to the characteristics of this kind of treatment, focused on the integration of information provision and the promotion of physical activity $[40,89]$, which would seem to favour an increase of mood in stressful situations. In discussing these results, we must consider the assessment tool used in the studies (i.e., the Profile of Mood States; [90]) that analysed different dimensions of the mood (e.g., Stress-Anxiety, Depression-Discomfort, etc.).

Undoubtedly, depression, anxiety and mood—especially if in comorbidity—are bound to impact the quality of life. Therefore, this meta-analysis also investigated the direct impact of therapies on quality of life.

A clinical effect (ES $=0.32$ ) on the quality of life was found, but it was also characterised by a higher heterogeneity. Cognitive behavioural therapy showed stronger effects compared to psycho-educational therapy and supportive-expressive therapy. Both the increase of self-awareness and the development of a non-judgmental attitude can promote better acceptance of the breast cancer diagnosis, and these effects could improve prognosis. Moreover, a more significant therapeutic efficacy in the improvement of the quality of life was observed in treatments made by more than one provider.

Generally, the meta-analysis showed results on the effectiveness of the different approaches on the outcomes considered; however, the high heterogeneity and the low quality of the studies highlighted the low robustness of these results and underlined the importance of developing studies with a better methodological structure. However, the findings of the present study allow us to suggest the development of a treatment including different professional figures, providing a specific role in the therapeutic process to implement the intervention in its different phases. Moreover, a clear definition of the activity of the control groups should also be considered in structuring a well-defined intervention protocol.

The group treatments included in the present study appeared to be more effective than individual ones in the improvement of the psychological outcomes that we have considered [35].

Overall, our findings allow us to conclude that both cognitive behavioural and psycho-educational therapies are associated with significant benefits. These treatments improve the quality of life and the mood, reducing anxiety and depression in women with breast cancer. These results agree with those observed by other studies $[35,91]$ which underlined the effectiveness of group therapy in women with localised breast cancer. These findings could be due to a preference of the patients to enter group therapy, which is characterised by a higher perceived social support.

Another reflection could be made on the choice of some studies to include health education (i.e., information about cancer and treatment and its side-effects) as a sort of "placebo" in the control groups $[25,59,62,63]$. The positive effect of this type of intervention could have affected the effect size of the analysis. However, the reception of educational materials did not show the same beneficial effect of structured psycho-educational programs, because it is not characterised by a group intervention but by the individual acquisition of notion about the illness condition [92]. 
This meta-analysis also underlined the confounding effect given by the use of different measures for the assessment of the analysed outcomes. This could have influenced the results of the studies. All the studies used self-reported measures of the outcomes, with the relative limits related to the objectivity of the information. However, there are few direct comparisons of the efficacy of these instruments in the population with breast cancer. Anxiety in people with cancer is commonly assessed through screening questionnaires [93]. Frequently, the analysed studies used the Hospital Anxiety and Depression Scale and the State-Trait Anxiety Inventory (see Table 1). The HADS appears to be more effective than other instruments for the assessment of anxious symptoms in patients with cancer. Conversely, the STAI was used as a screening tool and appeared to be more accurate in the measure of the longitudinal trend of the anxiety [93]. For this reason, the use of the STAI could be recommended for the screening of anxiety. Furthermore, it could be useful to analyse the effectiveness of the intervention in the follow-up. Many self-reported measures are commonly used to assess the depressive symptomatology in patients with breast cancer (see Table 1), although there is a need to identify the elective measure to better define depression in patients with breast cancer. Many investigations on depression in patients with cancer have used the HADS. These studies were mainly focused on identifying the cut-off scores for the diagnosis of depression in patients with cancer [94], even if the HADS represents a self-reported measure explicitly developed for assessing depression in patients within an outpatient medical setting [94]. The use of many instruments for the assessment of the psychological outcomes related to breast cancer and the different dimensions evaluated by the various questionnaires underlines the need to define standardised measures for the analysis of these psychological aspects in this clinical condition. A standardised assessment protocol could help to evaluate the effectiveness of the psychological interventions and could allow better comparison of the efficacy of the different types of psychological therapies.

Treatment duration from six to twelve weeks, compared to briefer or longer interventions, appeared to achieve better results for all the psychological dimensions considered in the present study. This finding is particularly relevant in the clinical setting, and it could also be useful from an economic point of view because a relatively brief psychological treatment could help to reduce psychopathological comorbidity in women with breast cancer and it could improve adherence to medical treatments, with lower direct and indirect costs than long-term treatments.

An interesting result of the present study is related to the good efficacy of cognitive behavioural therapy. Although many studies have shown the effectiveness of this type of intervention in different types of health promotion (for a review see [95]), the higher power of this approach in this meta-analysis could be due to the inclusion of studies used Mindfulness-Based Cognitive Therapy (MBCT). MBCT is an approach that integrates classical cognitive behavioural strategies with relaxation exercises for stress reduction and exercises of body perception. Previous studies found an effect of this therapy in promoting quality of life in patients [96-98] and survivors $[53,99]$ of cancer. Further studies are necessary to analyse the differences in the efficacy between standard cognitive behavioural therapies and mindfulness-based cognitive therapy. Moreover, our study considered only a few specific psychological approaches, but further studies could consider the benefits of other types of therapies [88]. For example, it has been shown that hypnosis-based psychotherapy is beneficial to reduce fatigue and cancer-related pain and to control sleep diseases [100-102]. Moreover, psychodynamic psychotherapy has resulted in a short-term improvement in patients' depressive symptoms [103], and interventions based on physical activity seem to improve health in cancer survivors [104]. For these reasons, further studies should consider other therapeutic approaches to define their effectiveness.

\section{Limitations}

The current review and meta-analysis has several limitations. The high heterogeneity of the studies represents the main weakness. The efficacy of the treatments and the comparison of the different approaches showed high variability among the studies. This heterogeneity influenced the power of the studies and the effect size concerning the efficacy of psychological treatments. The heterogeneity of 
studies, which represents the most impactful limitation of this work, makes generalisation of the results difficult. Most of the studies included a sample with patients at different cancer stages (e.g., stage I, metastatic stage), or with different medical interventions (e.g., chemotherapy, radiotherapy)—all aspects that could influence the psychological outcomes, modulating the effectiveness of the treatment itself. This could suggest a methodological reflection about the lack of a standard protocol in psychological treatments aimed at patients with breast cancer. It could guarantee higher transparency and replicability of the results, according to the results of the qualitative assessment, which showed some concerns about the risk of bias in the studies. This heterogeneity could be due to the choice to not have strictly defined inclusion criteria. This decision was influenced by the fact that this is the first meta-analysis pointed to compare the most common psychological interventions carried out in women with breast cancer. Second, it should be noted that grey literature (i.e., the unpublished studies showing different results) was excluded from this review, and this choice could represent a bias. Although the examination of the follow-up was not one of the objectives of our study, the systematic analysis showed high variability in the timing of follow-up assessments of the interventions. For further research, it could be interesting to analyse the long-term efficacy of the treatments, with a stricter definition of inclusion criteria and studies focused on follow-ups. Another possible selection bias was due to the exclusive consideration of group therapies without a comparison with individual ones.

Finally, the medical treatment of breast cancer has significantly changed in recent years, with the advent of new systemic treatments [105]. According to these therapeutic changes, psychological therapies have also changed.

This review suggests that psycho-educational, supportive-expressive and cognitive behavioural therapies are the gold-standard treatments for women with breast cancer. This conclusion is supported by their roles in the treatment of perseverative thinking, attentional biases and metacognitive processes $[105,106]$ that are involved in determining the outcomes analysed in the present systematic review and meta-analysis.

\section{Conclusions}

This review and meta-analysis confirms the efficacy of cognitive behaviour and psycho-educational therapies in both the modification of maladaptive cognitive schemas and the improvement of affective and emotional states. These treatments are of clinical importance to reduce anxiety and depression and to improve mood and quality of life in women with breast cancer.

Further studies should control methodological features and should structure a protocol able to enhance internal validity and provide more reliable findings.

Despite these limits, this review and meta-analysis could guide the development of new psychological interventions or help to optimize the efficacy of existing ones aimed at improving perceived well-being and quality of life.

Author Contributions: Conceptualisation, A.G. and M.C.; methodology, C.P. and I.B.; formal analysis, G.F., F.F.; investigation, C.P. and F.F.; data curation, A.G., M.C., C.P., I.B., G.F. and F.F.; writing-original draft preparation, A.G., M.C., C.P., I.B., G.F. and F.F.; writing-review and editing, A.G., F.F., G.F. and M.C.; supervision, M.C. and A.G. All authors have read and agreed to the published version of the manuscript.

Funding: Founding for Research from Ph.D. program in Psychology and Cognitivse Science. Department of Psychology, Sapienza Univeristy of Rome (Italy).

Acknowledgments: This work was not supported by any grant.

Conflicts of Interest: The authors declare that the research was conducted in the absence of any commercial or financial relationships that could be construed as a potential conflict of interest.

\section{References}

1. DeSantis, C.; Ma, J.; Bryan, L.; Jemal, A. Breast cancer statistics. CA Cancer J. Clin. 2014, 64, 52-62. [CrossRef] [PubMed] 
2. McGuire, S. World cancer report Geneva, Switzerland: World Health Organization, international agency for research on cancer, WHO Press, 2015. Adv. Nutr. 2016, 7, 418-419. [CrossRef] [PubMed]

3. Stagl, J.M.; Bouchard, L.C.; Lechner, S.C.; Blomberg, B.B.; Gudenkauf, L.M.; Jutagir, D.R.; Glück, S.; Derhagopian, R.P.; Carver, C.S.; Antoni, M.H. Long-term psychological benefits of cognitive-behavioral stress management for women with breast cancer: 11-year follow-up of a randomized controlled trial. Cancer 2015, 121, 1873-1881. [CrossRef] [PubMed]

4. Rojas, K.; Stuckey, A. Breast cancer epidemiology and risk factors. Clin. Obstet. Gynecol. 2016, 59, 651-672. [CrossRef] [PubMed]

5. Howlader, N.; Noone, A.M.; Krapcho, M.; Miller, D.; Bishop, K.; Altekruse, S.F.; Mariotto, A. SEER cancer statistics review, 1975-2013, National Cancer Institute. Bethesda 2016, 1-7.

6. Klein, D.; Mercier, M.; Abeilard, E.; Puyraveau, M.; Danzon, A.; Dalstein, V.; Velten, M. Long-term quality of life after breast cancer: A French registry-based controlled study. Breast Cancer Res. Treat. 2011, 129, 125-134. [CrossRef]

7. Koch, L.; Bertram, H.; Eberle, A.; Holleczek, B.; Schmid-Höpfner, S.; Waldmann, A.; Arndt, V. Fear of recurrence in long-term breast cancer survivors-Still an issue. Results on prevalence, determinants, and the association with quality of life and depression from the Cancer Survivorship-A multi-regional population-based study. Psycho-Oncology 2014, 23, 547-554. [CrossRef]

8. Gallagher, J.; Parle, M.; Cairns, D. Appraisal and psychological distress six months after diagnosis of breast cancer. Br. J. Health Psychol. 2002, 7, 365-376. [CrossRef]

9. Zabora, J.; BrintzenhofeSzoc, K.; Curbow, B.; Hooker, C.; Piantadosi, S. The prevalence of psychological distress by cancer site. Psycho-Oncology 2001, 10, 19-28. [CrossRef]

10. Kissane, D.W.; Ildn, J.; Bloch, S.; Vitetta, L.; Clarke, D.M.; Smith, G.C.; McKenzie, D.P. Psychological morbidity and quality of life in Australian women with early-stage breast cancer: A cross-sectional survey. Med. J. Aust. 1998, 169, 192-196. [CrossRef]

11. Fann, J.R.; Thomas-Rich, A.M.; Katon, W.J.; Cowley, D.; Pepping, M.; McGregor, B.A.; Gralow, J. Major depression after breast cancer: A review of epidemiology and treatment. Gen. Hosp. Psychiatry 2008, 30, 112-126. [CrossRef] [PubMed]

12. Tang, L.; Fritzsche, K.; Leonhart, R.; Pang, Y.; Li, J.; Song, L.; Fischer, I.; Koch, M.; Wuensch, A.; Mewes, R.; et al. Emotional distress and dysfunctional illness perception are associated with low mental and physical quality of life in Chinese breast cancer patients. Health Qual. Life Outcomes 2017, 15, 231. [CrossRef] [PubMed]

13. Galiano-Castillo, N.; Ariza-García, A.; Cantarero-Villanueva, I.; Fernández-Lao, C.; Díaz-Rodríguez, L.; Arroyo-Morales, M. Depressed mood in breast cancer survivors: Associations with physical activity, cancer-related fatigue, quality of life, and fitness level. Eur. J. Oncol. Nurs. 2014, 18, 206-210. [CrossRef] [PubMed]

14. Matsuda, A.; Yamaoka, K.; Tango, T.; Matsuda, T.; Nishimoto, H. Effectiveness of psychoeducational support on quality of life in early-stage breast cancer patients: A systematic review and meta-analysis of randomized controlled trials. Qual. Life Res. 2014, 23, 21-30. [CrossRef]

15. Denieffe, S.; Gooney, M. A meta-synthesis of women's symptoms experience and breast cancer. Eur. J. Cancer 2011, 20, 424-435. [CrossRef]

16. Antoni, S.; Ferlay, J.; Soerjomataram, I.; Znaor, A.; Jemal, A.; Bray, F. Bladder cancer incidence and mortality: A global overview and recent trends. Eur. Urol. 2017, 71, 96-108. [CrossRef]

17. Cohee, A.A.; Adams, R.N.; Fife, B.L.; Von Ah, D.M.; Monahan, P.O.; Zoppi, K.A.; Cella, D.; Champion, V.L. Relationship Between Depressive Symptoms and Social Cognitive Processing in Partners of Long-Term Breast Cancer Survivors. Oncol. Nurs. Forum 2017, 44, 44-51. [CrossRef]

18. Schmidt, M.E.; Wiskemann, J.; Steindorf, K. Quality of life, problems, and needs of disease-free breast cancer survivors 5 years after diagnosis. Qual. Life Res. 2018, 27, 2077-2086. [CrossRef]

19. Fiszer, C.; Dolbeault, S.; Sultan, S.; Brédart, A. Prevalence, intensity, and predictors of the supportive care needs of women diagnosed with breast cancer: A systematic review. Psycho-Oncology 2014, 23, 361-374. [CrossRef]

20. Cobeanu, O.; David, D. Alleviation of Side Effects and Distress in Breast Cancer Patients by Cognitive-Behavioral Interventions: A Systematic Review and Meta-analysis. J. Clin. Psychol. Med. 2018, 25, 335-355. [CrossRef] 
21. Font, A.; Rodríguez, E. Eficacia de las intervenciones psicológicas en mujeres con cáncer de mama. Psicooncología 2007, 4, 423.

22. Ye, M.; Du, K.; Zhou, J.; Zhou, Q.; Shou, M.; Hu, B.; Jiang, P.; Dong, N.; He, L.; Liang, S.; et al. A meta-analysis of the efficacy of cognitive behavior therapy on quality of life and psychological health of breast cancer survivors and patients. Psycho-Oncology 2018, 27, 1695-1703. [CrossRef]

23. Lantheaume, S.; Montagne, M.; Shankland, R. Intervention focused on resources to reduce anxiety and depression disorders in cancer patients: A pilot study. L'Encephale 2019. [CrossRef]

24. Kissane, D.W.; Bloch, S.; Smith, G.C.; Miach, P.; Clarke, D.M.; Ikin, J.; McKenzie, D. Cognitive-existential group psychotherapy for women with primary breast cancer: A randomised controlled trial. Psycho-Oncology 2003, 12, 532-546. [CrossRef]

25. Antoni, M.H.; Lechner, S.C.; Kazi, A.; Wimberly, S.R.; Sifre, T.; Urcuyo, K.R.; Carver, C.S. How stress management improves quality of life after treatment for breast cancer. J. Consult. Clin. Psychol. 2006, 74, 1143. [CrossRef]

26. Keefe, F.J. Cognitive behavioral therapy for managing pain. Clin. Psychol. 1996, 49, 4-5.

27. Classen, C.C.; Kraemer, H.C.; Blasey, C.; Giese-Davis, J.; Koopman, C.; Palesh, O.G.; Morrow, G.R. Supportive-expressive group therapy for primary breast cancer patients: A randomized prospective multicenter trial. Psycho-Oncology 2008, 17, 438-447. [CrossRef]

28. Ochoa, C.; Casellas-Grau, A.; Vives, J.; Font, A.; Borràs, J.M. Positive psychotherapy for distressed cancer survivors: Posttraumatic growth facilitation reduces posttraumatic stress. Int. J. Clin. Health Psychol. 2017, 17, 28-37. [CrossRef]

29. Wu, P.H.; Chen, S.W.; Huang, W.T.; Chang, S.C.; Hsu, M.C. Effects of a Psychoeducational Intervention in Patients with Breast Cancer Undergoing Chemotherapy. Nurs. Res. 2018, 20, 266-279. [CrossRef]

30. Ho, R.T.; Fong, T.C.; Lo, P.H.; Ho, S.M.; Lee, P.W.; Leung, P.P.; Chan, C.L. Randomized controlled trial of supportive-expressive group therapy and body-mind-spirit intervention for Chinese non-metastatic breast cancer patients. Support. Care Cancer 2016, 24, 4929-4937. [CrossRef]

31. Cipolletta, S.; Simonato, C.; Faccio, E. The effectiveness of psychoeducational support groups for women with breast cancer and their caregivers: A mixed methods study. Front. Psychol. 2019, 10. [CrossRef] [PubMed]

32. Dolbeault, S.; Cayrou, S.; Bredart, A.; Viala, A.L.; Desclaux, B.; Saltel, P.; Dickes, P. The effectiveness of a psycho-educational group after early-stage breast cancer treatment: Results of a randomized French study. Psycho-Oncology 2009, 18, 647-656. [CrossRef] [PubMed]

33. Chujo, M.; Mikami, I.; Takashima, S.; Saeki, T.; Ohsumi, S.; Aogi, K.; Okamura, H. A feasibility study of psychosocial group intervention for breast cancer patients with first recurrence. Support. Care Cancer 2005, 13, 503-514. [CrossRef] [PubMed]

34. Fillion, L.; Gagnon, P.; Leblond, F.; Gélinas, C.; Savard, J.; Dupuis, R.; Larochelle, M. A brief intervention for fatigue management in breast cancer survivors. Cancer Nurs. 2008, 31, 145-159. [CrossRef] [PubMed]

35. Naaman, S.C.; Radwan, K.; Fergusson, D.; Johnson, S. Status of psychological trials in breast cancer patients: A report of three meta-analyses. Psychiatry 2009, 72, 50-69. [CrossRef]

36. Jassim, G.A.; Whitford, D.L.; Hickey, A.; Carter, B. Psychological interventions for women with non-metastatic breast cancer. Cochrane Database Syst. Rev. 2015, 5. [CrossRef]

37. Fukui, S.; Kugaya, A.; Okamura, H.; Kamiya, M.; Koike, M.; Nakaniski, T.; Imoto, S.; Kanagawa, K.; Uchitomi, Y. A psychosocial group intervention for Japanese women with primary breast carcinoma. Cancer 2000, 89, 1026-1036. [CrossRef]

38. Phillips, K.M.; Antoni, M.H.; Lechner, S.C.; Blomberg, B.B.; Llabre, M.M.; Avisar, E.; Carver, C.S. Stress management intervention reduces serum cortisol and increases relaxation during treatment for nonmetastatic breast cancer. Psychosom. Med. 2008, 70, 1044-1049. [CrossRef]

39. Matthews, H.; Grunfeld, E.A.; Turner, A. The efficacy of interventions to improve psychosocial outcomes following surgical treatment for breast cancer: A systematic review and meta-analysis. Psycho-Oncology 2007, 26, 593-607. [CrossRef]

40. Chow, K.M.; Chan, J.C.; Choi, K.K.; Chan, C.W. A review of psychoeducational interventions to improve sexual functioning, quality of life, and psychological outcomes in gynecological cancer patients. Cancer Nurs. 2016, 39, 20-31. [CrossRef] 
41. Myrhaug, HT.; Mbalilaki, J.A.; Lie, N.K.; Hansen, T.; Nordvik, J.E. The effects of multidisciplinary psychosocial interventions on adult cancer patients: A systematic review and meta-analysis. Disabil. Rehabil. 2018, 1-9. [CrossRef] [PubMed]

42. Johannsen, M.; Farver, I.; Beck, N.; Zachariae, R. The efficacy of psychosocial intervention for pain in breast cancer patients and survivors: A systematic review and meta-analysis. Breast Cancer Res. Treat. 2013, 138, 675-690. [CrossRef] [PubMed]

43. Zhang, M.; Huang, L.; Feng, Z.; Shao, L.; Chen, L. Effects of cognitive behavioral therapy on quality of life and stress for breast cancer survivors: A meta-analysis. Min. Med. 2017, 108, 84-93. [CrossRef]

44. Liberati, A.; Altman, D.G.; Tetzlaff, J.; Mulrow, C.; Gøtzsche, P.C.; Ioannidis, J.P.; Moher, D. The PRISMA statement for reporting systematic reviews and meta-analyses of studies that evaluate health care interventions: Explanation and elaboration. PLoS Med. 2009, 6, e1000100. [CrossRef] [PubMed]

45. Moher, D.; Liberati, A.; Tetzlaff, J.; Altman, D.G.; Prisma Group. Reprint-Preferred reporting items for systematic reviews and meta-analyses: The PRISMA statement. Phys. Ther. 2009, 89, 873-880. [CrossRef] [PubMed]

46. Higgins, J.P.; Altman, D.G.; Gøtzsche, P.C.; Jüni, P.; Moher, D.; Oxman, A.D.; Sterne, J.A. The Cochrane Collaboration's tool for assessing risk of bias in randomised trials. BMJ 2011, 343, d5928. [CrossRef]

47. Cohen, J. Statistical Power Analysis for the Behavioral Sciences, 2nd ed.; Erlbaum: Hillsdale, NJ, USA, 1988.

48. Sidik, K.; Jonkman, J.N. A comparison of heterogeneity variance estimators in combining results of studies. Stat. Med. 2007, 26, 1964-1981. [CrossRef]

49. Higgins, J.P.; Thompson, S.G.; Deeks, J.J.; Altman, D.G. Measuring inconsistency in meta-analyses. BMJ 2003, 327, 557-560. [CrossRef]

50. Groarke, A.; Curtis, R.; Kerin, M. Cognitive-behavioural stress management enhances adjustment in women with breast cancer. Br. J. Health Psychol. 2013, 18, 623-641. [CrossRef]

51. Jang, S.H.; Kang, S.Y.; Lee, H.J.; Lee, S.Y. Beneficial effect of mindfulness-based art therapy in patients with breast cancer-A randomized controlled trial. Explore 2016, 12, 333-340. [CrossRef]

52. Johannsen, M.; O'Connor, M.; O'Toole, M.S.; Jensen, A.B.; Højris, I.; Zachariae, R. Efficacy of mindfulness-based cognitive therapy on late post-treatment pain in women treated for primary breast cancer: A randomized controlled trial. J. Clin. Oncol. 2016, 34, 3390-3399. [CrossRef] [PubMed]

53. Lengacher, C.A.; Reich, R.R.; Paterson, C.L.; Ramesar, S.; Park, J.Y.; Alinat, C.; Jacobsen, P.B. Examination of broad symptom improvement resulting from mindfulness-based stress reduction in breast cancer survivors: A randomized controlled trial. J. Clin. Oncol. 2016, 34, 2827. [CrossRef] [PubMed]

54. Reich, R.R.; Lengacher, C.A.; Alinat, C.B.; Kip, K.E.; Paterson, C.; Ramesar, S.; Budhrani-Shani, P. Mindfulness-based stress reduction in post-treatment breast cancer patients: Immediate and sustained effects across multiple symptom clusters. J. Pain. Symptom Manag. 2017, 53, 85-95. [CrossRef] [PubMed]

55. Zhang, J.Y.; Zhou, Y.Q.; Feng, Z.W.; Fan, Y.N.; Zeng, G.C.; Wei, L. Randomized controlled trial of mindfulness-based stress reduction (MBSR) on posttraumatic growth of Chinese breast cancer survivors. Psychol. Health Med. 2017, 22, 94-109. [CrossRef]

56. Kenne Sarenmalm, E.; Martensson, L.B.; Andersson, B.A.; Karlsson, P.; Bergh, I. Mindfulness and its efficacy for psychological and biological responses in women with breast cancer. Cancer Med. 2017, 6, 1108-1122. [CrossRef] [PubMed]

57. Kissane, D.W.; Grabsch, B.; Clarke, D.M.; Smith, G.C.; Love, A.W.; Bloch, S.; Li, Y. Supportive-expressive group therapy for women with metastatic breast cancer: Survival and psychosocial outcome from a randomized controlled trial. Psycho-Oncology 2007, 16, 277-286. [CrossRef] [PubMed]

58. Bower, J.E.; Crosswell, A.D.; Stanton, A.L.; Crespi, C.M.; Winston, D.; Arevalo, J.; Ganz, P.A. Mindfulness meditation for younger breast cancer survivors: A randomized controlled trial. Cancer 2015, 121, 1231-1240. [CrossRef]

59. Lechner, S.C.; Whitehead, N.E.; Vargas, S.; Annane, D.W.; Robertson, B.R.; Carver, C.S.; Antoni, M.H. Does a community-based stress management intervention affect psychological adaptation among underserved black breast cancer survivors? J. Natl. Cancer Inst. 2014, 50, 315-322. [CrossRef]

60. Dodds, S.E.; Pace, T.W.; Bell, M.L.; Fiero, M.; Negi, L.T.; Raison, C.L.; Weihs, K.L. Feasibility of Cognitively-Based Compassion Training (CBCT) for breast cancer survivors: A randomized, wait list controlled pilot study. Support Care Cancer 2015, 23, 3599-3608. [CrossRef] 
61. Boyle, C.C.; Stanton, A.L.; Ganz, P.A.; Crespi, C.M.; Bower, J.E. Improvements in emotion regulation following mindfulness meditation: Effects on depressive symptoms and perceived stress in younger breast cancer survivors. J. Consult. Clin. Psychol. 2017, 85, 397. [CrossRef]

62. Bordeleau, L.; Szalai, J.P.; Ennis, M.; Leszcz, M.; Speca, M.; Sela, R.; Pritchard, K.I. Quality of life in a randomized trial of group psychosocial support in metastatic breast cancer: Overall effects of the intervention and an exploration of missing data. J. Clin. Oncol. 2003, 21, 1944-1951. [CrossRef] [PubMed]

63. McKiernan, A.; Steggles, S.; Guerin, S.; Carr, A. A controlled trial of group cognitive behavior therapy for Irish breast cancer patients. J. Psychosoc. Oncol. 2010, 28, 143-156. [CrossRef] [PubMed]

64. Lee, H.; Lim, Y.; Yoo, M.S.; Kim, Y. Effects of a nurse-led cognitive-behavior therapy on fatigue and quality of life of patients with breast cancer undergoing radiotherapy: An exploratory study. Cancer Nurs. 2011, 34, E22-E30. [CrossRef] [PubMed]

65. Björneklett, H.G.; Lindemalm, C.; Rosenblad, A.; Ojutkangas, M.L.; Letocha, H.; Strang, P.; Bergkvist, L. A randomised controlled trial of support group intervention after breast cancer treatment: Results on anxiety and depression. Acta Oncol. 2012, 51, 198-207. [CrossRef]

66. Gudenkauf, L.M.; Antoni, M.H.; Stagl, J.M.; Lechner, S.C.; Jutagir, D.R.; Bouchard, L.C.; Avisar, E. Brief cognitive-behavioral and relaxation training interventions for breast cancer: A randomized controlled trial. J. Consult. Clin. Psychol. 2015, 83, 677. [CrossRef]

67. Cohen, M.; Fried, G. Comparing relaxation training and cognitive-behavioral group therapy for women with breast cancer. Res. Soc. Work Pract. 2007, 17, 313-323. [CrossRef]

68. Spielberger, C.D. State-Trait anxiety inventory. In The Corsini Encyclopedia of Psychology; John Wiley \& Son's, Inc.: Hoboken, NJ, USA, 2010; p. 1.

69. Zigmond, A.S.; Snaith, R.P. The hospital anxiety and depression scale. Acta Psychiatr. Scand. 1983, 67, 361-370. [CrossRef]

70. Morey, L.C. Personality Assessment Inventory: Professional Manual; Psychological Assessment Resources: Tampa, FL, USA, 1991.

71. Beck, A.T.; Ward, C.; Mendelson, M.; Mock, J.; Erbaugh, J. Beck depression inventory (BDI). Arch. Gen. Psychiatry 1961, 4, 561-571. [CrossRef]

72. Roberts, R.E.; Vernon, S.W. The Center for Epidemiological Studies Depression Scale: Its use in a community sample. Am. J. Psychiatry 1983, 140, 41-46.

73. Cella, D.F.; Tulsky, D.S.; Gray, G.; Sarafian, B.; Linn, E.; Bonomi, A.; Eckberg, K. The Functional Assessment of Cancer Therapy scale: Development and validation of the general measure. J. Clin. Oncol. 1993, 11, 570-579. [CrossRef]

74. Moriwaki, S.Y. The Affect Balance Scale: A validity study with aged samples. J. Gerontol. 1974, $29,73-78$. [CrossRef] [PubMed]

75. Derogatis, L.R.; Melisaratos, N. The brief symptom inventory: An introductory report. Psych. Med. 1983, 13, 595-605. [CrossRef]

76. Shacham, S. A shortened version of the Profile of Mood States. J. Pers. 1983, 47, 305-306. [CrossRef] [PubMed]

77. Aaronson, N.K.; Ahmedzai, S.; Bergman, B.; Bullinger, M.; Cull, A.; Duez, N.J.; Kaasa, S. The European Organization for Research and Treatment of Cancer QLQ-C30: A quality-of-life instrument for use in international clinical trials in oncology. JNCI 1993, 85, 365-376. [CrossRef]

78. Stewart, A.L.; Hays, R.D.; Ware, J.E. The MOS short-form general health survey. Reliability and validity in a patient population. Med. Care 1998, 26, 724-735. [CrossRef]

79. Shim, E.J.; Mehnert, A.; Koyama, A.; Cho, S.J.; Inui, H.; Paik, N.S.; Koch, U. Health-related quality of life in breast cancer: A cross-cultural survey of German, Japanese, and South Korean patients. Breast Cancer Res. Treat. 2006, 99, 341-350. [CrossRef]

80. Sheard, T.; Maguire, P. The effect of psychological interventions on anxiety and depression in cancer patients: Results of two meta-analyses. Br. J. Cancer 1999, 80, 1770. [CrossRef]

81. Devine, E.C.; Westlake, S.K. The effects of psychoeducational care provided to adults with cancer: Meta-analysis of 116 studies. Oncol. Nurs. Forum 1995, 22, 1369-1382.

82. Kaczkurkin, A.N.; Foa, E.B. Cognitive-behavioral therapy for anxiety disorders: An update on the empirical evidence. Dialogues Clin. Neurosci. 2015, 17, 337. 
83. Johnson, J.A.; Rash, J.A.; Campbell, T.S.; Savard, J.; Gehrman, P.R.; Perlis, M.; Garland, S.N. A systematic review and meta-analysis of randomized controlled trials of cognitive behavior therapy for insomnia (CBT-I) in cancer survivors. Sleep. Med. Rev. 2016, 27, 20-28. [CrossRef]

84. Shannonhouse, L.; Myers, J.; Barden, S.; Clarke, P.; Weimann, R.; Forti, A.; Porter, M. Finding your new normal: Outcomes of a wellness-oriented psychoeducational support group for cancer survivors. JSGW 2014, 39, 3-28. [CrossRef]

85. Jafari, A.; Goudarzian, A.H.; Nesami, M.B. Depression in women with breast cancer: A systematic review of cross-sectional studies in Iran. APJCP 2018, 19, 1. [PubMed]

86. Driessen, E.; Hollon, S.D. Cognitive behavioral therapy for mood disorders: Efficacy, moderators and mediators. Psychiatr. Clin. 2010, 33, 537-555. [CrossRef] [PubMed]

87. Mohr, D.C.; Boudewyn, A.C.; Goodkin, D.E.; Bostrom, A.; Epstein, L. Comparative outcomes for individual cognitive-behavior therapy, supportive-expressive group psychotherapy, and sertraline for the treatment of depression in multiple sclerosis. J. Consult. Clin. Psychol. 2001, 69, 942. [CrossRef]

88. Boutin, D.L. Effectiveness of cognitive behavioral and supportive-expressive group therapy for women diagnosed with breast cancer: A review of the literature. JSGW 2007, 32, 267-284. [CrossRef]

89. Ohlsson-Nevo, E.; Andershed, B.; Nilsson, U. Psycho-educational intervention on mood in patients suffering from colorectal and anal cancer: A randomized controlled trial. Nord. J. Nurs. Res. 2017, 37, 135-142. [CrossRef]

90. McNair, D.M.; Lorr, M.; Droppleman, L.F. Manual for the Profile of Mood States; Educational Industrial Testing Service: San Diego, CA, USA, 1971.

91. Bellver-Pérez, A.; Peris-Juan, C.; Santaballa-Beltrán, A. Effectiveness of therapy group in women with localized breast cancer. Int. J. Clin. Health Psychol. 2019, 19, 107-114. [CrossRef]

92. Fawzy, F.I.; Fawzy, N.W.; Wheeler, J.G. A post-hoc comparison of the efficiency of a psychoeducational intervention for melanoma patients delivered in group versus individual formats: An analysis of data from two studies. Psycho-Oncology 1996, 5, 81-89. [CrossRef]

93. Stark, D.P.H.; House, A. Anxiety in cancer patients. Br. J. Cancer 2000, 83, 1261. [CrossRef]

94. Trask, P.C. Assessment of depression in cancer patients. JNCI Monogr. 2004, 2004, 80-92. [CrossRef]

95. Hofmann, S.G.; Asnaani, A.; Vonk, I.J.; Sawyer, A.T.; Fang, A. The efficacy of cognitive behavioral therapy: A review of meta-analyses. Cognit. Ther. Res. 2012, 36, 427-440. [CrossRef] [PubMed]

96. Haller, H.; Winkler, M.M.; Klose, P.; Dobos, G.; Kuemmel, S.; Cramer, H. Mindfulness-based interventions for women with breast cancer: An updated systematic review and meta-analysis. Acta Oncol. 2017, 56, 1665-1676. [CrossRef] [PubMed]

97. Park, E.S.; Kim, S.J.; Kim, S.I.; Chun, Y.J.; Lee, P.S.; Kim, H.J.; Han, K.S. A study of factors influencing health promoting behavior and quality of life in the elderly. J. Korean Acad. Nurs. 1998, 28, 638-649. [CrossRef]

98. Rosen, K.D.; Paniagua, S.M.; Kazanis, W.; Jones, S.; Potter, J.S. Quality of life among women diagnosed with breast Cancer: A randomized waitlist controlled trial of commercially available mobile app-delivered mindfulness training. Psycho-Oncology 2018, 27, 2023-2030. [CrossRef] [PubMed]

99. Gonzalez-Hernandez, E.; Romero, R.; Campos, D.; Burychka, D.; Diego-Pedro, R.; Baños, R.; Cebolla, A. Cognitively-Based Compassion Training $\left(\mathrm{CBCT}^{\circledR}\right)$ in Breast Cancer Survivors: A Randomized Clinical Trial Study. Integr. Cancer Ther. 2018, 17, 684-696. [CrossRef] [PubMed]

100. Grégoire, C.; Nicolas, H.; Bragard, I.; Delevallez, F.; Merckaert, I.; Razavi, D.; Vanhaudenhuyse, A. Efficacy of a hypnosis-based intervention to improve well-being during cancer: A comparison between prostate and breast cancer patients. BMC Cancer 2018, 18, 677. [CrossRef] [PubMed]

101. Mendoza, M.E.; Capafons, A.; Jensen, M.P. Hypnosis attitudes: Treatment effects and associations with symptoms in individuals with cancer. Am. J. Clin. Hypn. 2017, 60, 50-67. [CrossRef] [PubMed]

102. Téllez, A.; Rodríguez-Padilla, C.; Martínez-Rodríguez, J.L.; Juárez-García, D.M.; Sanchez-Armass, O.; Sánchez, T.; Jaime-Bernal, L. Psychological effects of group hypnotherapy on breast cancer patients during chemotherapy. Am. J. Clin. Hypn. 2017, 60, 68-84. [CrossRef]

103. Beutel, M.E.; Fischbeck, S.; Binder, H.; Blettner, M.; Brähler, E.; Emrich, K.; Zeissig, S.R. Depression, anxiety and quality of life in long-term survivors of malignant melanoma: A register-based cohort study. PLoS ONE 2015, 10, e0116440. [CrossRef] 
104. Maxwell-Smith, C.; Zeps, N.; Hagger, M.S.; Platell, C.; Hardcastle, S.J. Barriers to physical activity participation in colorectal cancer survivors at high risk of cardiovascular disease. Psycho-Oncology 2017, 26, 808-814. [CrossRef]

105. Beatty, L.; Kemp, E.; Butow, P.; Girgis, A.; Schofield, P.; Turner, J.; Koczwara, B. A systematic review of psychotherapeutic interventions for women with metastatic breast cancer: Context matters. Psycho-Oncology 2018, 27, 34-42. [CrossRef] [PubMed]

106. Ehring, T.; Watkins, E.R. Repetitive negative thinking as a transdiagnostic process. Int. J. Cogn. Ther. 2008, 1, 192-205. [CrossRef]

(C) 2020 by the authors. Licensee MDPI, Basel, Switzerland. This article is an open access article distributed under the terms and conditions of the Creative Commons Attribution (CC BY) license (http://creativecommons.org/licenses/by/4.0/). 